\title{
Demographic change and pension reform in Spain: an assessment in a two-earner, OLG model
}

\author{
Alfonso R. Sánchez Martín \\ U. Pablo de Olavide
}

\author{
Virginia Sánchez Marcos \\ Universidad de Cantabria and Fedea
}

June 4, 2010

\begin{abstract}
Recent pension reforms in Spain have been guided by two opposite goals: achieving financial stability and improving redistributive aspirations. In particular, reforms implemented in 1997/2001 entailed a mixture of both through (i) changes in the pension formula, (ii) the extension of the entitlement to early retirement to all cohorts, and, finally, (iii) increases in survival pensions. This paper builds an Applied General Equilibrium OLG model that captures the fundamental non-stationarity of the Spanish reality (ageing population, education transition and an increasing female attachment to the labour market) to assess the impact of those reforms. As a novel feature with respect to the literature, households in our model economy are made up of two potential earners that make saving and labour supply decisions. Our main conclusions from the analysis are at three different levels. First, the Spanish pension system is clearly unsustainable, with the pension expenditure reaching a figure of about $18 \%$ of the GDP in 2050, and the reforms have been clearly ineffective in improving the pension system's financial prospects. Second, the reforms have had substantial redistribution effects, benefiting low educated groups against high educated and future cohorts against current cohorts. Finally, we show that exploring the financial prospects with traditional single earner households models may result in underestimates of the future financial burden of the pension system.
\end{abstract}

JEL codes: D58, H55, J11 


\section{Introduction}

The aging of the population and the imminent retirement of the large cohorts of "Baby Boomers" have generated widespread concern about the financial sustainability of PAYG pension systems. In response, most countries have engaged in reforms of their current systems, largely aimed at reducing pension generosity. ${ }^{2}$ Spain has not been an exception to this general trend, having introduced significant changes in its pension rules from 1997 to 2002 . The Spanish reform, however, stands out as remarkably timid and unambitious, packing together a heterogeneous mix of measures with opposite financial consequences. This outcome is probably the result of two principal forces. On the one hand, the Spanish pension system enshrines the principle of solidarity as one of its core foundational values. ${ }^{3}$ This reflects in the existence of important redistributive dispositions in a system whose basic structure is largely "Bismarkian" (ie. individual pensions are proportional to previous contributions). Redistribution is essentially achieved by establishing minimum guaranteed benefits for both old-age and survival pensions. ${ }^{4}$ Furthermore, survival pensions themselves are considered a key redistributive tool, by making it possible to bridge the large gender gap in pension income currently existing in Spain (stemming from the extremely low participation rates of the females belonging to Spanish most senior cohorts). On the other hand, the recent economic performance of the Spanish economy (prior to the 2008 crisis) has been quite remarkable, featuring a particularly good behavior of the labor market and large flows of immigrants. Naturally, this has produced a marked improvement in the short-term financial indicators of the pension system, which may have led Spanish legislators to be mistaken about the real long-term health of the system. All in all, the political process has delivered a fragmentary set of reforms with clearly contradicting financial consequences. Fiscal consolidation has been pursued by increasing the length of the averaging period in the pension formula and by imposing stronger penalties for an insufficient record of contributions. At the same time and for the sake of enhanced solidarity, the generosity of survival pensions has been significantly increased and dispositions limiting the right to early retirement (before 65) to some specific cohorts have been waived.

The goal of this paper is to assess the impact of the set of implemented reforms, both in financial and in redistributive terms (ie. changes in welfare). To achieve this goal we develop a dynamic general equilibrium model specially designed to capture the specificities of the Spanish case. Individuals in the model differ in their gender, education and age of birth. Upon entering the labor market they are grouped together into households, which take life-cycle consumption/savings decisions and labor participation decisions. The head of household is assumed to participate continuously, until an endogenously chosen retirement age. The spouse, in contrast, makes a once and for all decision on labor participation at the beginning of her productive life. This setting is rich enough to accommodate most of the essential elements of the question in hand: a detailed reproduction of old-age pensions (including certain features that are contingent on household composition, such as, for instance, the size of the minimum pension) and survival pensions, the changing patterns in education and female labor participation by cohort and the endogenous reaction in both labor and savings to changes in the institutional environment.

\footnotetext{
${ }^{2} \mathrm{~A}$ concise review of recent reforms can be found in Whiteford and Whitehouse (2006) or (in far greater detail) in Caseay et al. (2003).

${ }^{3}$ See Objetivo 1 in MTAS (2005)

${ }^{4}$ In the interval 2001-2007, $28 \%$ of old-age pensions and $37 \%$ of survival pensions were increased up to the annually legislated minimums. Note also that some redistribution takes place through the existence of Special Regimes (eg. self-employed, farmers, sailors, coal miners ...).
} 
The literature on general equilibrium analysis of pensions in the context of an ageing population has relied on standard OLG models in the Auerbach and Kotlikoff (1987) tradition. The literature is rather large, having explored issues like the intra-generational redistribution implied by the pension system (eg. Cubeddu (1996) emphasizes differences in education, sex and race while Huggett and Ventura (1999) explore the role of minimum pensions); the insurance provided against survival risks or idiosyncratic income shocks (eg. Storesletten et al. (1999) or İmrohoroğlu et al. (1995)) or the strong inter-generational consequences of pension reform or even complete privatization (eg. De Nardi et al. (1999)). In the Spanish case, Conesa and Garriga (2001) study the inter-generational properties of the Spanish pension system in a representative agent life-cycle model. Rojas (2005) extends the analysis to a framework in which productivity depends on age and Sánchez (2010) to a framework in which individuals are ex ante heterogenous in their education and select their retirement age endogenously. Finally, Díaz-Giménez and Díaz-Saavedra (2009) introduce earnings shocks that individuals suffer throughout their lives. However, up to now, this literature has been based upon single-earner household decision makers, playing down the changing pattern of female labor participation and sometimes ignoring survival pensions altogether.

By reflecting gender differences and having households made of two potential earners as the economic units of our model we join a strand of the macroeconomic literature that initially focused on the determinants of the increase in female labour supply and then moved towards the analysis of its aggregate consequences. Some outstanding examples of the former are Caucutt et al. (2002), Greenwood et al. (2005), Olivetti (2006) or Attanasio et al. (2008). The contributions that emphasize the importance of two potential earner households for policy analysis are particularly close to our work. For example, Guner et al. (2008) find that married women account for a disproportionately large fraction of the changes in labour supply (hours and participation) resulting from tax reforms. The aggregate implications of reforms can, then, vary wildly depending on whether a single-earner or a double-earner household is used.

Intrahousehold heterogeneity models have also made headway in pension analysis. There has been a steady progress in modeling the interaction of pension and fiscal rules and household decisions in partial equilibrium. For example, Scholz et al. (2006) focus on the optimal savings behaviour of US couples, taking explicit account of the role of survival pension benefits. Gustman and Steinmeier (2000) and Casanova (2009) focus on the joint retirement decisions made by the household's members. Finally, a two-earner household framework has been used in a general equilibrium setting in Kaygusuz (2008). He studies the steady state implications of several reforms of the US pension system, like the elimination of the spouse's benefit or changes in the progressive character of pension benefits. Our work differs from his in a number of dimensions (eg. we reproduce population ageing and other non-stationary patterns of the economy, endogenize retirement and allow for gender differences in mortality risk). To the best of our knowledge, the present paper is the first application of household models in a general equilibrium analysis of pension reform with population aging.

Our two-potential-earner households model is carefully calibrated to reproduce the basic demographic and economic properties of the Spanish economy as of the beginning of the twenty-first century. In particular, we reproduce the institutional details of the old-age and survival pension system and the observed historical changes in education and female labor supply. The model's unobservable parameters are selected to reproduce the aggregate macroeconomic and labor-market performance in the interval 2001-2007. Once equipped with a fully specified model we undertake several experiments. We simulate the model in its current form to generate a precise quantitative projection of the finances of the Spanish pension system in the interval 2010-2050. We then study the financial and welfare consequences 
of the 1997/2001 reforms and, finally, emphasize the importance of including survival pensions and gender differences in the model by comparing with the predictions obtained from a model that only features old-age pensions. Our findings can be summarized as follows:

- In its current form, the Spanish pension system is clearly unsustainable. This is so even in the presence of strong immigration flows and a steady improvement in the employment rates of the economy (they increase from 64 to $79 \%$ ). Pension expenditure as a proportion of GDP will more than double by 2050, reaching $18 \%$ from an initial value of $7 \%$. Survival pensions also roughly double in the interval and so contribute $15 \%$ of the total increase. The total implicit unfunded liability of the system is calculated to add up to $105.4 \%$ of the tax base in 2001 (using a $5 \%$ discount factor). Under these circumstances, any feasible fiscal path will demand strong tax hikes. In particular, keeping the overall budget balanced year by year by adjusting the income tax would demand a tax rate jump from 13 to $21 \%$ in 2050 .

- The figures above leave no doubt about the ineffectiveness of the implemented reforms in tackling the financial imbalances of the system. Notwithstanding this, they have had some financial impact. If we exclude the extension of the early retirement entitlement, the implemented reforms have reduced the implicit unfunded liabilities (as a proportion of the tax base) by around 9 percentage points. Including the extension of early retirement, however, we find the opposite result: an increase of around 11 percentage points. Note, however, that the final total expenditure to GDP ratio would have been roughly the same even in the most favorable scenario.

- The reforms had quite appreciable welfare consequences, even after allowing for behavioral responses (which are appreciable, especially in terms of retirement). In comparison with the status quo, they redistribute the cost of ageing by putting a larger burden on more educated vs less educated individuals and on the cohorts of current active workers vs future cohorts. Whether the household has a working spouse makes little difference if the legal retirement ages are left unaltered. Remarkably, the decision to extend the right to retire early to all cohorts generated an appreciable redistribution from all other groups to the current and future low-educated households with a working female.

- Finally, we repeat our base simulation but in an economy without survival pensions. To make the experiment meaningful, we recalibrate the old-age pension so that we roughly reproduce the observed total pension expenditure to GDP ratio in 2002/2007. The simulated equilibrium path obtained is surprisingly different, projecting significantly smaller aggregate pension expenditures in the long term. The underestimation peaks at around 2050 with a divergence of as much as 1.5 percentage points (or a $10 \%$ error in the pension expenditure to GDP ratio). The implicit unfunded liabilities of the system are assessed to be 7 percentage points smaller than in the benchmark case.

The rest of the paper is organized as follows. In Section 2 we describe the model that serves as the basic tool for our analysis. In Section 3.1 we explain the details of the demographic patterns included in the simulations, while Section 3.2 deals with the calibration of the unobservable parameters of the model and of the details of the pension system. In Section 3.3 we check the ability of the model to achieve its calibration targets and test its performance in other non-calibrated dimensions. The main findings of the paper are reported in sections 4.1 (projection of the economy in its current form), 4.2 (projections under the pre $1997 / 2001$ reforms system) and 4.3 (impact of omitting the survival pensions). The paper closes with some general comments in section 5. Additional information is confined to several appendices. 


\section{The model}

The model consists of overlapping generations of ex-ante heterogenous agents that live up to $I$ periods in an economy without aggregate uncertainty. However, at the micro level, individuals are uncertain about the length of their life. There is no insurance market for this risk and borrowing against future pension income is not allowed. Concerning the degree of openness of the model, we allow for migrant flows, but we abstract from international financial relationships. A period in the model stands for one year of real time, which we denote by $t$ when referring to calendar time and by $i$ when referring to age. The cohort the individual belongs to is denoted by $u(u=t-i+1)$. We use the subindex $g$ to distinguish between the household members: $g=1$ denotes the head of the household, while $g=2$ refers to a second potential wage earner, that (for the sake of simplicity) we identify with the female of the house. After the entry into the labor market (at the age of 20), all individuals are grouped in households and start making economic decisions. At that time, households are classified according to their educational attainment into one of $J$ possible categories (denoted by $j \in J=\{1, \ldots, J\}$ ). As a general notational convention, variables characterizing the household or its members are written in lower case and indexed by (depending on the context) education, gender, year of birth, age or calendar time. Aggregate variables are denoted with capital letters and are normally indexed by calendar time.

\subsection{Demography}

Demography evolves in accordance with a one sex population model, where individuals are classified according to their country of birth as "Natives", $N^{t}$, or "Migrants", $M^{t}{ }^{5}$ The number of people born at $t$ is determined by the profile of age-specific fertility rates $\left\{\theta_{i}^{t}\right\}$ (assumed to vary between the threshold ages $f_{0}$ and $f_{1}$ ):

$$
N_{1}^{t}=\sum_{i=f 0}^{f 1} \theta_{i}^{t}\left(N_{i}^{t-1}+M_{i}^{t-1}\right)
$$

After-birth population dynamics is given by:

$$
N_{i}^{t}=h s_{i}^{t-i+1} N_{i-1}^{t-1} \quad M_{i}^{t}=h s_{i}^{t-i+1} M_{i-1}^{t-1}+F_{i}^{t} \quad 1<i \leq I
$$

where $F_{i}^{t}$ stands for immigrant flows and $\left\{h s_{i}^{u}\right\}_{i=1}^{I}$ for the cohort-u vector of age-conditional survival probabilities. Equations (1) and (2) constitute the law of motion of the population in the interval $t \in\left(t_{0}, t_{1}\right)$, characterized by a demographic transition in which the fertility and mortality parameters are changing in time (see section 3.1). After $t_{1}$ (set to 2050 in the simulations) the fertility and mortality patterns stay constant and immigration flows progressively die out. After $t_{2}=t_{1}+I$ the age-distribution of the population is constant (the population becomes stable), and we finally assume the convergence of the entire economy to a final balance growth path at time $t_{3} .{ }^{6}$ The complete time span of the simulation is represented by $\mathcal{T}$.

\footnotetext{
${ }^{5}$ The household formation process for migrants older that 20 years is assumed to reproduce the distribution in the population at large. That is to say, for each possible education level and year of birth of a newly arrived immigrant we assume the same distribution of single and biparental families in the corresponding household as in the entire population. This assumption is not realistic, but has a negligible impact on our results.

${ }^{6}$ The final balance growth is only really reached asymptotically, but in the simulation it is assumed to be reached in period $t_{3}=2220$. We check that the particular value chosen for $t_{3}$ does not affect the performance of the economy in the interval of interest $\left(t_{0}, t_{1}\right)$.
} 


\subsection{The public sector}

The main role played by the Public Sector is to run a Defined-Benefit pension system, financed with the contributions made by active workers (ie, run on a PAYG basis). The social contributions paid in calendar time $t$ by a worker of age $i$, are a fixed proportion, $\varsigma$, of his/her covered earnings, $\operatorname{cov}_{i}^{t}$. Covered earnings, in turn, are also a fixed proportion, $1-\chi$, of the individual gross labor income, $w^{t} \varepsilon_{i, g}$, with an annually legislated maximum $C_{M}^{t}$ :

$$
\operatorname{cov}_{i}^{t}=\min \left\{(1-\chi) w^{t} \varepsilon_{i}^{t}, C_{M}^{t}\right\}
$$

$\varepsilon_{i, g}$ is the endowment of efficient labor units at age $i$, and $w^{t}$ is the market price of one unit of efficient labor. The contributive wedges $\chi$ are the product of several legal dispositions. On the one hand, some components of the overall remuneration do not generate pension rights (eg. travel expenses, food tokens and other subsistence expenses). On the other hand (and more importantly) there are appreciable differences in the treatment of covered earnings across the different Social Security schemes. While we can expect a rather small difference between gross income and covered earnings in the General Regime (RGSS), the situation is very different for other pension regimes. ${ }^{7}$ As we do not explicitly model the different Social Security schemes, the wedges make it possible to reproduce the average proportionality between income and covered earnings in the data.

Elegible workers (ie. those with a long enough contributive record, $h \geq 15$ ) can claim an old-age pension at any time after the early retirement age, $\tau_{m}$, following a complete withdrawal from the labor force. The initial pension for an individual belonging to cohort $u$ who retires at age $\tau$ after $h$ years of contributions is:

$$
b(\tau, h, u)=\alpha^{E}(\tau) \alpha^{H}(h)\left(\frac{\sum_{e=\tau-D}^{\tau-1} c o v_{e}^{u+e-1}}{D}\right)
$$

The formula combines a moving average of covered earnings in the $D$ years immediately preceding retirement (called benefit base) and two linear replacement rates:

- The replacement rate, $\alpha^{E}(\tau)$ captures early retirement penalties. For each year that the individual anticipates retirement (from the Normal Retirement Age, $\tau_{N}$, of 65), the final benefit is reduced by a $\Delta \alpha^{E}$ percent. This penalty also depends on the length of the contributive record (see section 3.2.1). There is also a annual bonus $\Delta \alpha_{+65}^{E}$ for staying employed after 65. Formally:

$$
\alpha^{E}(\tau)= \begin{cases}\alpha_{0}^{E} & \text { if } \tau<60 \\ \alpha_{0}^{E}+\Delta \alpha^{E}(\tau-60) & \text { if } \tau \in\{60, \ldots, 64\} \\ 1.0+\Delta \alpha_{+65}^{E}(\tau-65) & \text { if } \tau \geq 65\end{cases}
$$

- The replacement rate $\alpha^{H}(h)$ captures penalties associated with a record of social contributions shorter than 35 years, with the penalty being more severe for those with shorter records $\left(\Delta \alpha_{1}^{H}>\Delta \alpha_{2}^{H}\right)$ :

$$
\alpha^{H}(h)= \begin{cases}\alpha_{0}^{H}+\Delta \alpha_{1}^{H}(h-15) & \text { if } h \in\{15, \ldots, 25\} \\ \alpha_{1}^{H}+\Delta \alpha_{2}^{H}(h-25) & \text { if } h \in\{25, \ldots, 35\} \\ 1.0 & \text { if } h \geq 35\end{cases}
$$

\footnotetext{
${ }^{7}$ Self-employed workers (covered by the Regimen Especial de Trabajadores Autónomos, RETA) can decide on the size of their declared covered earnings. As a result, the vast majority opt to contribute the legal minimum for most of their careers (see eg. Boldrin, Jiménez, and Peracchi (2004)).
} 
The initial pension $b(\tau, h, u)$ is indexed to price inflation and subject to annually legislated maximums, $b M^{t}$, and minimums, $b m^{t}$, (whose value changes in the presence of a dependant spouse). Therefore, the effective pension income in year $t$ for the individual above would be:

$$
i b_{i}^{t}(\tau)=\min \left\{b M^{t}, \max \left\{b m^{t}, b(\tau, h, t-i+1)\right\}\right\}
$$

Along with the old-age pensions, the system also provides survival pensions to the widows/widowers of deceased spouses. The initial value is obtained as a fraction $\alpha^{V}$ of the benefit base of the deceased. We simplify the complexity of the real world eligibility conditions by assuming that all surviving spouses older than $\tau_{m}^{V}$ qualify for the survival pension. ${ }^{8}$ There is also a specific guaranteed minimum for survival pensions, denoted by $b m_{V}^{t}$. All in all, the pension income of a survival spouse is:

$$
i v_{i}^{t}=\max \left\{b m_{V}^{t}, \alpha^{V} b(\tau, t-i+1, h)\right\}
$$

In addition to running the pension system, the Public Sector performs two additional functions: it runs a fiscal system and it incurs a certain amount of public expenditure, $C P^{t} .{ }^{9}$ Both tasks are extremely simplified in our model. Fiscal revenue is assumed to originate in the full taxation of involuntary bequests and from a proportional tax levied on both labor and capital income, while public expenditure is a fixed proportion of aggregate output.

\subsection{The household problem}

Households are the economic agents of the model. At the onset, they are all made up of two people (i.e. we assume no heterogeneity in the marital status) and a number of children that varies with the cohort. Households make decisions about their labour supply and about their optimal life cycle profiles of consumption and asset holdings $\left(c_{i}^{t}\right.$ and $a_{i}^{t}$ respectively, with $t=u+i-1$ ). In particular, labour supply decisions take two forms: a life-time female participation decision, $E^{u}=\{0,1\}$, and a coordinated retirement decision by the active members of the household, $\tau^{u} \in\{60, \ldots 70\} .^{10}$ Male participation along the life-cycle is, therefore, assumed exogenous, with the exception of the age of withdrawal from the labor force. ${ }^{11}$

We assume both spouses share the same age of birth, $u$, and educational attainment, $j .{ }^{12}$ Besides education and age of birth, we introduce as an additional source of heterogeneity the

\footnotetext{
${ }^{8}$ The age requirement is a proxy for the real requirement in terms of number of years of contributions.

${ }^{9} C P^{t}$ stands for both the running cost of the public administrations and for expenditure in the provision of public goods. For simplicity, we abstract from the welfare impact of the consumption of those goods.

${ }^{10}$ Our assumption on female labor supply is supported by the evidence in Adam (1996), describing a dual participation behavior by Spanish women. Although most (young) women participate at early ages, the population clearly divides into two groups after childbirth, with those who fail to reenter shortly after the event ending up in a withdrawal from the labor market. This polar conduct has been confirmed by Gutiérrez-Domenech (2005) in the Spanish case, and contrasted with the patterns observed in other countries. The assumption of simultaneous retirement of husband and wife draws on the strong empirical correlation found between the two decisions both in Spain and in other countries (see eg. Jiménez et al. (1999) for the Spanish cases, and Banks et al. (2007) for US and UK).

${ }^{11}$ In our view, the two margins of the household labor supply that are more sensitive to economic incentives are the female participation decisions and the retirement decision of the head. The latter is particularly important in our context because of its large sensitivity to the incentives provided by the pension system. Note, in particular, that the size of the pension benefits obtained by a household depends strongly on its composition and the labor participation of its members.

${ }^{12}$ This simplifying assumption implies an overstatement of the degree of assortative mating in the economy, which, in Spain, is around $70 \%$ for low educated workers.
} 
utility cost that the household incurs if the female participates in the labour market throughout her lifetime. ${ }^{13}$ We represent this extra heterogeneity with the distribution function $\Phi$, which is assumed to be independent of the year of birth or the education of the spouses. Households are formed at the age of entry into the labor market (20 years of age) and suffer no changes in their composition other than those derived from the effects of mortality. As the mortality hazard takes its toll on the progressively older family members, widows and widowers arise in the economy. In order to account for the heterogeneity in household size that arises due to mortality we make the following simplification assumption. We assume that household income at a particular age, conditional on education and age of birth, is a weighted sum of the first and second potential earners' incomes. The weight for each g-earner is equal to the fraction of families in which the g-earner is still alive. Obviously, the income of the second potential earner is contingent on the female participation decision.

We model the female participation decision as a trade-off between the extra labor income generated for the household, the specific utility cost for the household, $z$, (an extraction from $\Phi$ ), and a direct financial cost (representing the purchase in the market of services that would have otherwise been generated by the spouse). As a consequence, there is a fraction of couples with a working spouse for each cohort $u$ and education level $j$.

As usual, each household behavior is obtained from the solution of an intertemporal optimal control problem. Preferences are represented by a pure-time-impatience discount factor $\beta<1$ and a period utility function $U(c, E, \tau, z)$ that depends on consumption, on leisure (ie. retirement age, $\tau$, and female's participation, $E$ ) and on the utility cost of participating, $z$. The problem of the household belonging to cohort $u$ (omitting the educational type to simplify notation) is:

$$
\begin{array}{cl}
E^{u}, \tau^{u},\left\{c_{i}^{t}, a_{i}^{t}\right\}_{i=1}^{I} & \sum_{i=1}^{I} \beta^{i-1} s_{i}^{u} U\left(c_{i}^{t}, E^{u}, \tau^{u}, z\right) \\
& c_{i}^{t}-C_{i}^{t} E^{t}+a_{i+1}^{t+1}=H I_{i}^{t}+\left(1+\bar{r}^{t}\right) a_{i}^{t} \\
& a_{1}^{u}=0 \quad a_{I}^{u+I-1}=0 \\
& a_{i}^{u+i-1} \geq 0 \quad \forall i \geq \tau
\end{array}
$$

where $s_{i}^{u}$ stands for the survival probability (of at least one household member) to age $i$, $\bar{r}^{t}$ stands for the net-of-taxes return on capital (ie. $\bar{r}^{t}=r^{t}\left(1-\varphi^{t}\right)$, with income tax rate $\left.\varphi^{t}\right) . C_{i}^{t}$ capture the direct financial costs of female labor market participation, which varies across cohorts depending on the average number of children raised by the household and on market wages. The household income $\left(H I_{i}^{t}\right)$ is the sum of the contributions made by each of the potencial family earners $W_{i, g}^{t}$, with $g=\{1,2\}$. Before retirement, the net labor income of member $g$ takes the form:

$$
W_{i, g}^{t}=\left(1-\varphi^{t}\right)\left[w^{t} \varepsilon_{i g}^{t}-\varsigma \operatorname{cov}_{i, g}^{t}\right]
$$

Gross labor income $w^{t} \varepsilon_{i g}^{t}$ is the product of the endowment of efficient labor units and their market price, while payroll taxes are a fixed proportion of covered earnings (defined in eq. (3)). All in all, the household income is:

\footnotetext{
${ }^{13}$ The introduction of this type of heterogeneity to model participation decision is also used in Guner et al. (2008) and Kaygusuz (2009).
} 


$$
H I_{i}^{t}=\pi_{i, 1}^{t} W_{i, 1}^{t}+\pi_{i, 2}^{t} W_{i, 2}^{t} E^{t}
$$

where $\pi_{i, g}^{t}$ is the proportion of families that, despite the effects of mortality, still include the g-earner at age $i$.

Similarly, for retirees we can write household income as:

$$
H I_{i}^{t}=\pi_{i, 1}^{t} \xi_{i, 1}^{t} B_{i, 1}^{t}+\pi_{i, 2}^{t} \xi_{i, 2}^{t} B_{i, 2}^{t}
$$

where $B_{i, g}^{t}$ is the sum of the old-age and survival pensions of member $g$ at age $i$ (computed in accordance with expressions (7) and (8)) and $\xi_{i, g}^{t}$ is the share of members of gender $g$ of cohort $u=t-i+1$ that are entitled to get pensions in accordance with legal dispositions (discussed in section 2.2).

\subsection{Technology}

The production side of the model is entirely neoclassical: we assume a standard technology, with a constant returns to scale production function, $F(K, L)$, no adjustment costs and exogenous labor-augmenting technological progress, $A^{t}$. The growth rate of labor productivity, $\rho$, is exogenous and constant.

\subsection{Equilibrium}

An equilibrium path over the time interval $\mathcal{T}$ is a set of time series of population aggregates and distributions, household decisions (consumption, savings and labor supply), aggregate inputs, prices and public policies (tax rates, public consumption and minimum and maximum pensions and contributions) with the standard properties: households are rational (ie., they solve the problem in (9) taking the environment as given), factor markets clear, prices are competitive, the public budget balances and an aggregate feasibility constraint is observed. Appendix A provides a formal definition of the equilibrium of the model economy. As in the standard Auerbach and Kotlikoff (1987) methodology, we assume the convergence of the equilibrium path to a final balance growth path in finite time. Nevertheless, the initial equilibrium is non-stationary (as in eg. (Kotlikoff et al. 2000)).

\section{Calibration}

We calibrate our model to mimic the observable economic and demographic characteristics of the Spanish economy, and in accordance with standard projections for future demographic and productivity trends. The next two sections review the details of the demographic and economic calibrations. Section 3.3 tests the effectiveness of the calibration by comparing the model predictions and the empirical evidence in the interval 2001-2007.

\subsection{Demography}

A period in the model stands for one year of calendar time and we assume a maximum lifespan $I$ of 100 years. The simulated equilibrium path starts in $t_{0}=2001$. Our demographic model in section 2.1 is only used starting in 2008. For the interval 2001-2007 we reproduce the observed population aggregates, distribution and age profiles of fertility and survival probabilities. ${ }^{14}$

\footnotetext{
${ }^{14}$ Our source is the publicly available demographic information from the Spanish National Statistics Institute (INE). In particular, we use the 2001-Census, the "Explotación estadística del Padrón" database, and the mortality and fertility information in the "Movimiento Natural de la Población" records.
} 
From 2008 to 2050 we simulate a changing pattern of fertility and mortality, in accordance with the main scenario ("hipótesis 1") by the Spanish Statistical Institute (INE). ${ }^{15}$ The main patterns can be appreciated in figure 12 (confined to appendix B.1). The total fertility rate is assumed to recover from the extremely low values observed during the nineties (in 1995, 1.2 children per women nationwide) to a final stationary value of 1.53 in 2050 . We also reproduce the trend towards lower mortality rates by assuming that life expectancy rises from the 76.6 years observed in 2001 to 81.0 years in 2050 for males and from 83.4 to 87 years for females. In both cases, the bulk of the recovery is concentrated in the first decades of the simulation. For the immigration flows, we reproduce the observed data between 2000 and 2005 (which registered flows of unprecedented size for the Spanish historical experience) and follow INE ("hipótesis 1") for the projection in the interval 2005/2050. ${ }^{16}$

\subsection{Calibration of the Economic Model}

The model described in section 2 becomes an operative tool once we complete its calibration by assigning specific values to all its parameters. This involves three different processes. First, subsection 3.2.1 introduces a subset of parameters with reasonably clear empirical counterparts. Secondly, as our simulation starts directly with a non-stationary equilibrium (assimilated to the state of the economy in 2001) we need initial values for a number of predetermined. Again, clear candidates are available for this choice (section 3.2.2). Finally, all the other parameters (reflecting preferences, technology and the distribution of the heterogeneity in the economy) have to be calibrated, ie. they are set to reproduce some specific properties of the observed behavior of the Spanish economy in the interval 2001-2007 (section $3.2 .3)$.

\subsubsection{Parameters with empirical counterparts}

\section{Education, income and cost of the female participation decision}

Education. For compatibility with our income data, individuals are classified in three educational groups $(j \in J=\{1,2,3\})$. The lowest education level corresponds to those who fail to complete secondary education, the highest level is made of tertiary graduates and the medium level includes all the rest. The recent historical evolution of this variable, as measured in the Labour Force Survey, has been truly remarkable. Low educated workers were almost $95 \%$ of the total among the individuals born in 1920/1930. This figured was reduced to $55 \%$ for the cohort born in 1970/80. We reproduce this historical trend and assume it to continue at a progressively slower pace for the cohorts born after 1975 . The resulting time series of the evolution of the distribution by education are displayed in figure 13 in appendix B.2. This appendix also provides more details on the construction of the education distribution from the observable information.

Life-cycle profiles of labor earnings. The individual profiles of efficiency labor units are estimated using the microdata of the European Community Household Panel (ECHP) 1994-2001. We use standard regression techniques to obtain earnings profiles for males and females and for each of our educational levels. Note that the actual labor earnings enjoyed by

\footnotetext{
${ }^{15}$ Available from INE web page: "INEbase/ Proyecciones de población calculadas a partir del Censo de Población de 2001". e-Link: http://www.ine.es/metodologia/t20/t2030251h.htm.

${ }^{16}$ We use the 1997-2004 Encuesta de Variaciones Residenciales microdata to compute the net aggregate flows of incoming population and its breakdown by age. This breakdown is assumed constant throughout the simulation path 2005-2050. The initial stock of immigrants is taken from the 2001 Census data.
} 
the workers in the model change in time depending on the endogenous equilibrium wages and in agreement with an exogenous trend of productivity increases. At the aggregate level, our assumptions on the supply-side of the economy guarantee a constant share of labor income on the total GDP. The estimated profiles and a more detailed description of the calibration process is presented in appendix B.3. The properties of the profiles are standard: they increase with age and education and exhibit a gender gap of, on average, $28 \%$ of the earnings of the male. ${ }^{17}$ The relative endowment of efficiency labor units are subsequently held constant throughout the simulation. ${ }^{18}$ The individual profiles are combined within the household according to equation (11). Needless to say, these profiles are of paramount importance in our simulation. For instance, they largely determine the size of the pension benefits and exert a strong influence on the timing of retirement and on the female participation decisions (see appendix C).

Direct monetary costs of participation. The labor participation of the female inflicts monetary costs on the household that stem from the fixed costs of working, foregone home production, child care cost and others. This is reflected in our model with the cost process $C_{i}^{t}$. In each period, the cost per child is modelled as a fixed proportion, $\bar{c}$, of the annual gross labor income of the female of the household. Consequently, it changes across cohorts and education groups with the variation in the average number of children and with the changes in the market valuation of the time of the female of the household.

The measurement problems associated with those costs are, however, big, making the calibration of $\bar{c}$ challenging. ${ }^{19}$ Our approach is, then, to limit the direct monetary costs in the model to the (relatively well measured) average price of child care in those ages in which schooling is not covered by the public education system. ${ }^{20}$ According to Consumer (2005) the average monthly price of a nursery for children of up to two years of age is around $33 \%$ of the monthly earnings of a low educated female. Therefore we set the cost parameter $\bar{c}$ in our model to this value and assume the cost to be constant for the first fours years of the child's life (and zero for the remaining lifespan of the household). This is likely to be a lower bound of the real monetary cost, and we may in consequence be overestimating the utility cost, but the empirical performance of the resulting model (see section 3.2.3 below) is good. ${ }^{21}$

\footnotetext{
${ }^{17}$ Note that this is partially driven by gender differences in hours worked and in the incidence of unemployment.

${ }^{18} \mathrm{We}$ do not allow for changes in the relative productivity by age, by education or by gender (ie. there is no improvement in the estimated gender gap, conditional on age and education). However the average earnings gender gap in our model evolves endogenously according to changes in the composition of the female labour force. In the data there are several forces driving the evolution of the wage gender gap. On the one hand, as women work harder the gender gap goes down due to the returns to experience, but, on the other hand, the selection process that brought more women to the labor market may increase the gender gap (see Attanasio et al. (2008)). In our model economy, only the latter effect is present (via the heterogeneity in education).

${ }^{19}$ For example, children-related costs estimates based on expenditures of two-earner households may be affected by selection as argue by Hong (2008). In Spain there is a special concern in this respect as the role of grandparents taking care of their grandchildren while their parents work has been documented to be important, see Tobío (2003).

${ }^{20}$ As documented in INECSE (2004) children in Spain start attending the state-provided public school when they are four years old. Before that age, public or private nurseries or kindergarten have to be used in general (the public ones are actually in very low supply).

${ }^{21}$ An alternative strategy is to calibrate or estimate these costs within a structural model using as a source of identification data on female participation by the age and number of children as well as data on home production time allocation. However, this approach would require a more detailed specification of the female participation decision than the one we implement here.
} 


\begin{tabular}{ccccc}
\hline $\begin{array}{c}\text { Averaging } \\
\text { period }\end{array}$ & $\begin{array}{c}\text { Early retirement } \\
\text { penalties }\end{array}$ & $\begin{array}{c}\text { Contribution } \\
\text { penalties }\end{array}$ & $\begin{array}{c}\text { Legal } \\
\text { ages }\end{array}$ & $\begin{array}{c}\text { Survival } \\
\text { pension }\end{array}$ \\
\hline$D=15$ & $\alpha_{0}^{E}=\left\{\begin{array}{c}0.60(h \leq 30) \\
0.65(\text { other }) \\
0.7(h \geq 40)\end{array}\right.$ & $\alpha_{0}^{H}=0.5$ & $\begin{array}{c}\tau_{m}=61 \\
\tau_{N}=65\end{array}$ & $\alpha^{V}=0.52$ \\
$\Delta \alpha_{+65}^{E}=0.02$ & $\Delta \alpha^{H}=\left\{\begin{array}{l}3 \% h \in(15,25) \\
2 \% h \in(25,35)\end{array}\right.$ & \\
\hline
\end{tabular}

Table 1: Pension System parameters in the benchmark simulation. All notation is as in section 2.2 .

\section{Pension System}

The institutional parameters are set to capture the fundamental features of the Spanish social security system. Although it is not possible to achieve an exact matching with our stylized model, we can go a long way towards reproducing the main quantitative properties of the model. ${ }^{22}$ Our benchmark simulation corresponds to the structure in place after the latest implemented reforms in 1997 and 2002, and, in particular, to the institutional values observed in 2002 that are shown in table 1.

Additionally, we model the discretional components of the pension system (the annual values of minimum pensions and maximum contributions) as linear functions of the anual income per worker. The parameters of those functions are calibrated to reproduce the corresponding 2001 ratios:

$$
b m^{01} / \bar{W}^{01}=\left\{\begin{array}{c}
0.30 \quad \text { with dependant spouse } \\
0.35 \quad \text { without dependant spouse }
\end{array} \quad C_{M}^{01} / \bar{W}^{01}=1.76 \quad b m_{V}^{01} / \bar{W}^{01}=0.26\right.
$$

Note, finally, that some of the parameters of the system are without direct observable counterparts and have to be calibrated. First the model pay-roll tax, $\varsigma$, does not fit with the real-world contribution rate. The problem is that our contribution only finances pension obligations, while the real-world counterpart also provides insurance for health, disability and other contingencies. To proceed, we calibrate the value of $\varsigma$ to reproduce the average balance of the pension system (as a proportion of the annual GDP) in the interval 20012007. Panel (c) in table 2 shows both the target value (a positive imbalance slightly above $1 \%$ of the GDP) and the parameter value selected for the simulation (a rate equal to $12.1 \%$ of the pensionable income). Second, we calibrate the wedges separating gross income and covered earnings, $\chi_{j}$, through indirect inference by selecting the values that simultaneously reproduce (i) the ratio of the average pension to average productivity $(\bar{b} / \bar{y})$ in the interval

\footnotetext{
${ }^{22}$ The current pension system features several Regimes that differ to varying degrees (See Boldrin et al. (2004) for a complete description of the Spanish pension system). At the moment, $76 \%$ of the affiliated workers and $57 \%$ of the pensioners belong to the General Regime. A number of specific Regimes coexist with the General, but most of them will progressively disappear as part of the general overhaul of the system agreed in 1997 (Pacto de Toledo). In the future, the system will see the coexistence of the General Regime and the Self-employed Regime. Consequently, the model parameters are chosen to match those systems as far as possible. Fortunately, there are strong similarities between the pension formulas (old-age and survival), legal ages, eligibility conditions and minimum pensions in both systems. As discussed in section 2.2, the main difference lies in the determination of the covered earnings, which is reflected in the income wedges $\chi$.
} 
2001-2007; and (ii) the proportion of workers receiving the minimum pensions. The former statistic identifies the wedge for workers of medium and above medium education while the latter is associated with the wedge for low income workers. As reproduced in table 2, the calibrated wedges are rather substantial for those with medium and high education, but more moderate for the low educated type, who are the majority of the population. Finally, to add more realism to the model we allow the ratio of pensions per employee, $\phi$, to differ from one. ${ }^{23}$ This reflects some differences in the elegibility conditions among the Special Pension Regimes. More importantly, this is intended to alleviate our rigid assumptions on the lifecycle participation of individuals -especially women. ${ }^{24}$ To minimize the loss of discipline implied by this extra parameter we keep the value of $\phi$ constant by cohort and education. We calibrate it to the value that best reproduces the average coverage rate of the system $\left(B / P_{+65}\right.$ the total number of pensions per person age 65 or older $)$ in the interval 2001-2007. The resulting figure is 1.12 (table 2).

\subsubsection{Initial conditions}

As our simulation path starts in 2001, the values of a number of relevant variables are already predetermined by the decisions previously taken by the households. Using the notation of previous sections and denoting the life-span by $\mathcal{I}=\{20, \ldots, 100\}$ and the cohorts whose pension benefit is already known in 2001 by $\mathcal{U}$, the variables whose starting values are obtained empirically are the following ${ }^{25}$ :

- Asset holdings by age and education, $\left\{a_{i, j}^{01}\right\} i \in \mathcal{I} j \in J$ for all cohorts alive at the beginning of the simulation. In the model we reproduce the wealth to income ratios observed in the Bank of Spain's EFF (Encuesta Financiera de las Familias). Proceeding in this way, we get an aggregate capital to GDP ratio of 2.4 in 2001, very close to the value measured by BBVA-Ivie (2005) (2.3).

- Retirement ages of the more senior Spaniards, by education, $\left\{\tau_{j}^{u}\right\} \quad u \in \mathcal{U}, j \in J$. This information is obtained from the latest data release of the MCVL-08 (Muestra Continua de Vidas Laborales). ${ }^{26}$ When this is interacted with our other model assumptions we generate an aggregate average retirement age in 2001 of 62.7, very close (although slightly below) the empirical figure of 62.9 years.

- Pensions benefits of the more senior citizens, $\left\{B_{j, g}^{u}\right\} \quad u \in \mathcal{U}, j \in J, g \in\{1,2\}$. As with asset holdings, we reproduce in our model the ratio of old-age pensions (by cohort, education and gender) to the average wage in 2001, according to the information in MCVL-08. After aggregation, we generate a pension expenditure to GDP ratio $(P P / Y)$ slightly above the data: $6.94 \%$ vs $6.75 \%$.

- Female labor participation by education for cohorts born before 1970. We use the Spanish Labor Survey (EPA) to calculate the proportion of double-earner households by cohort $u<1971$ and education type $j \in J$; In our model (recall our assumption

\footnotetext{
${ }^{23}$ Note that the coefficients $\xi_{i, g}^{t}$ in the household income equation (eq (12) of section 2.3) are obtained by multiplying the employment rate by $\phi$.

${ }^{24}$ For instance, it is possible for a number of second-earners to remain inactive during large parts of their life cycle and reenter the labor force at very advanced ages, precisely to qualify for an old-age pension of their own. This type of behavior is not captured by the model.

${ }^{25}$ The original Auerbach and Kotlikoff (1987) approach was to take those values from an initial steady state. In this work, however, we follow more recent practices where, whenever possible, the predetermined variables are taken directly from the data.

${ }^{26}$ This is an extract from the archive of administrative records of the Social Security System.
} 
about the full life-cycle participation of a working female) this proportion identifies the participation rate of the females.

\subsubsection{Preference, technology and distributional parameters}

For the remaining parameters of the model we select values that cause the model to mimic the performance of some fundamental variables of the Spanish economy in the interval 20012007. As reflected in Table 2, the targeted properties capture the aggregate behavior of the pension system, the labor market and some basic macroeconomic ratios.

\section{Preferences}

As indicated in section 2.3, the period utility function at age $i, U_{i}(c, E, \tau, z)$, depends on consumption $c$ and leisure (ie. retirement age, $\tau$, and female participation decision, $E$ ). More specifically, we use a separable CES function:

$$
U_{i}=\frac{c^{1-\eta}}{1-\eta}-z E+\alpha I(i>\tau)
$$

with intertermporal elasticity of substitution (IES $=1 / \eta$ ), relative value of leisure $\alpha$ and utility cost of female participation $z$. These parameters are calibrated as follows:

The consumption component of the utility is modelled as a logarithm (IES=1), in accordance with econometric evidence (Hurd (1989) for US or Jiménez-Martín and Sánchez-Martín (2007) for Spain). It has the additional property that it is the only functional form that guarantees the invariance of the retirement decision in the presence of exogenous productivity growth. $\alpha$ is selected to reproduce the average retirement age (63.0). The best figure we get simulating the model is 62.7 , which is close to the empirical figure, but not entirely successful. This reflects the limitations derived from the existence of a relatively small number of households at each age. Note that, conditional on female labour market decision, the heterogeneity in the utility cost has no effect on the retirement decision of the head (due to the additive separability assumed in the utility function). ${ }^{27}$ The utility cost of a working-female is instrumental for the alignment of the employment rates of the model and of the data. We assume $z$ to be normally distributed across the members of each cohort. Two parameters, then, have to be calibrated: the mean $\left(\mu_{z}\right)$ and standard deviation $\left(\sigma_{z}\right)$ of the normal. They are chosen to guarantee a seamless transition from the predetermined participation rates of older cohorts, in particular those born before 1970 (directly obtained from the data), to the endogenous rates produced by the model for younger generations (those born after 1970). This is done in order to minimize the distance between the female participation rates by education in the model and in the data. The results are reported in panel (b) of table 2. Concerning participation rates of males, they are assumed to be constant throughout the simulated path and equal to their observed value in 2001 (83.3, 89.8 and 92.9 for low, medium and highly educated workers respectively). Finally, to complete the specification of individual preferences we choose a value for $\beta$ that makes our model reproduce the average 2001-2007 $K / Y$ ratio of the economy (as reported in table 2 ).

\footnotetext{
${ }^{27}$ This makes the computation of the equilibrium more tractable, but also results in a relatively inflexible aggregate retirement age.
} 


\begin{tabular}{lccc}
\hline \hline \multicolumn{4}{c}{ (a) Macro-Aggregates } \\
\hline Target & Data & Model & Parameter \& value \\
& & & \\
$K / Y$ & 2.40 & 2.42 & $\beta=0.985$ \\
$I / Y \%$ & 28.3 & 28.7 & $\delta=6.0 \%$ \\
$r k / Y \%$ & 32.6 & 32.6 & $\theta=0.326$ \\
$C P / Y \%$ & 14.8 & 14.8 & $c_{p}=0.148$ \\
Productivity growth $\%$ & & 1.7 & $\rho$
\end{tabular}

\section{(b) Labor Supply}

\begin{tabular}{|c|c|c|c|}
\hline Target & Data & Model & Parameter \& value \\
\hline Female participation* \% (Low educ) & 67.8 & 67.2 & \\
\hline (Medium education) & 76.5 & 75.8 & $\mu_{z}=1.07$ \\
\hline (High education) & 84.6 & 82.7 & $\sigma_{z}=0.67$ \\
\hline Average retirement age & 63.0 & 62.7 & $\alpha=0.75$ \\
\hline \multicolumn{4}{|c|}{ (c) Pension System } \\
\hline Target & Data & Model & Parameter \& value \\
\hline $\mathrm{PSB} / \mathrm{Y} \%$ & 1.08 & 1.12 & $\varsigma=12.1 \%$ \\
\hline Minimum pension coverage $(\mathrm{OA}) \%$ & 28.1 & 26.6 & $\chi_{l o w}=8 \%$ \\
\hline $\bar{b} / \bar{y}$ replacement rate & 20.86 & 21.3 & $\chi_{m e d}=\chi_{h i g h}=35 \%$ \\
\hline Number of Survival pensions & 2.14 & 2.15 & $\tau_{m}^{V}=45$ \\
\hline Pension coverage rate $\%$ & 88.5 & 86.7 & $\phi=1.12$ \\
\hline
\end{tabular}

Table 2: Calibrated parameter values and empirical averages in the interval 2001-2007. $*=$ Female labor force participation by members of the cohort born in $1970 . \quad \mathrm{OA}=$ Old Age pensions. 


\section{Technology}

Aggregate output is generated with a Cobb-Douglas production function, $Y=K^{\zeta} L^{1-\zeta}$. Therefore, the supply side of the model is completely specified by choosing the capital share in aggregate income $\zeta$, the rate of capital depreciation, $\delta$, and the constant productivity growth rate, $\rho$. Panel (a) in table 2 reproduces the macro-targets and the parameters employed to achieve them. $\zeta$ is set to reproduce the average capital income share in the interval 20012007, as measured in Puch and Licandro (1997). The depreciation rate is selected to mimic the average Investment-to-GDP ratio, while the exogenous productivity growth rate, $\rho$, is set to $1.7 \%$ to reproduce the long term value employed in the central scenario of EPC (2006). The final macro aggregate to be calibrated is the government expenditure to output ratio. In this case we simply use the average 2001-2007 value as an input in the model (parameter $c_{-} p$ ) and assume it to be constant throughout the equilibrium path.

\subsection{Testing the model: behaviour in 2001-2007}

We use the observable performance of the Spanish economy in the 2001-2007 interval to test the calibration of the model on those dimensions that have not been explicitly targeted. Graphs in figure 1 illustrate the time-series behaviour of both the real economy and our simulated equilibrium path in 2001-2007. The aggregate capital to GDP ratio, K/Y, is a good starting point, conveniently bundling the saving and labor supply decisions of the agents in the model (top-left graph in figure 1). The model reproduces the average level in the data, but fails to capture the intensity of the upward trend shown by the real world. This is not surprising, as the model is not built to reproduce the cyclical patterns of the economy. ${ }^{28}$ A similar phenomenon will reveal itself repeatedly in other variables subject to a strong cyclical character.

Given the target of our work, it is especially important to scrutinize the performance of the pension system. The top-right and bottom-left graphs of figure 1 show the aggregate pension expenditure and the pension system balance (difference of annual contributions and pension expenditures) expressed as a ratio to GDP (PP/Y and PSB/Y respectively). The degree of coincidence is, in our view, very satisfactory, both in terms of levels and trends.

In order to understand the driving forces of $\mathrm{PP} / \mathrm{Y}$ and the ability of the model to track them we decompose it as follows:

$$
\begin{aligned}
\frac{P P^{t}}{Y^{t}}=\left(\frac{\bar{b}^{t}}{\bar{y}^{t}}\right)\left(\frac{B^{t}}{E^{t}}\right) & =\left(\frac{\bar{b}^{t}}{\bar{y}^{t}}\right) \quad\left(\frac{B^{t}}{P_{+65}^{t}}\right) \quad\left(\frac{P_{+65}^{t}}{P_{20-64}^{t}}\right) \quad\left(\frac{P_{20-64}^{t}}{E^{t}}\right)= \\
& =\text { rep rate } * \text { cov rate } * \text { dep ratio } * 1 / \text { emp rate }
\end{aligned}
$$

Total pension expenditure is the product of the average pension, $\bar{b}^{t}$, and the number of pensioners, $B^{t}$. Similarly, we express the GDP as the product of the number of employees, $E^{t}$, times the average productivity per employee, $\bar{y}^{t}$. Then, trivial algebra makes it possible to express the $\mathrm{PP} / \mathrm{Y}$ ratio as the product of four factors: the replacement rate of the average pension in terms of average productivity, $\bar{b}^{t} / \bar{y}^{t}$, the coverage rate or number of pensions per person aged 65 or more, $B^{t} / P_{+65}^{t}$, a dependency ratio, $P_{+65}^{t} / P_{20-64}^{t}$, capturing demographics and, finally, the inverse of the employment rate $E^{t} / P_{20-64}^{t}$, reflecting the performance of the labor market. ${ }^{29}$ Figure 2 displays the evolution of the four factors in the interval 2001-2007.

\footnotetext{
${ }^{28}$ The cyclical variation has been particularly acute in the last phases of expansion and downturn in Spain. The expansion stage was mainly driven by housing investment and the low interest rates resulting from the ECB monetary policy and the arrival of large flows of foreign capital.

${ }^{29}$ In the notation of section 3.1, the total population of a particular age $i$ at a specific calendar time $t, P_{i}^{t}$, is the sum of the stocks of Native and Migrants of that age $P_{i}^{t}=N_{i}^{t}+M_{i}^{t}$.
} 


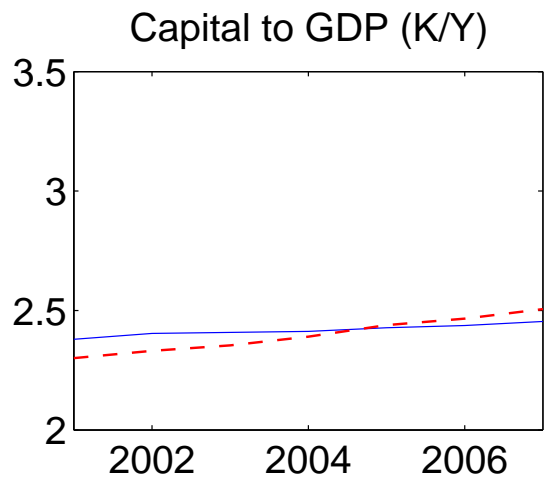

Pension Expenditure to GDP (PP/Y)
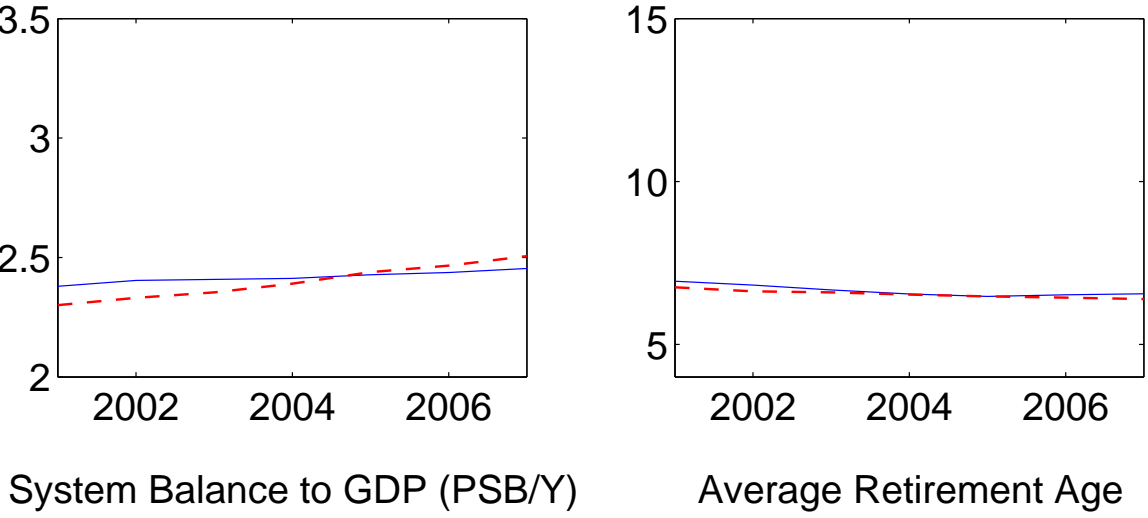

Average Retirement Age
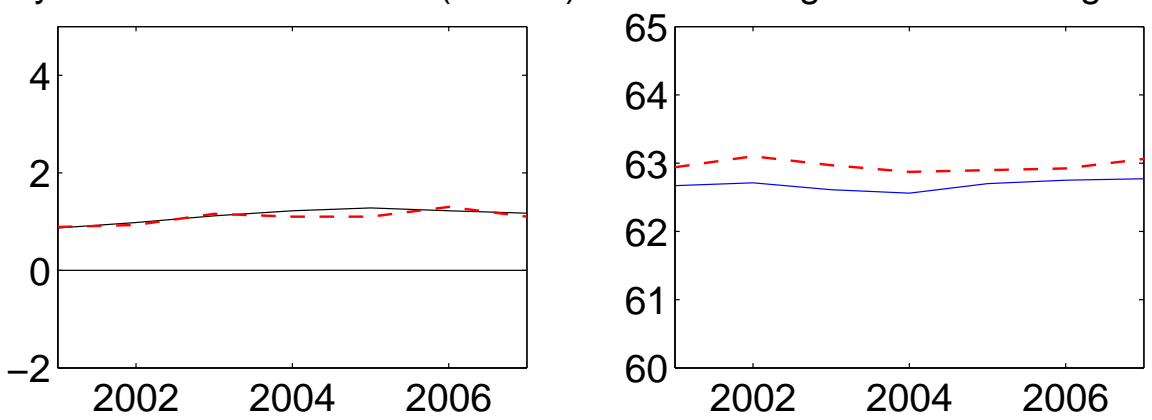

Figure 1: Model vs real world performance in 2001-2007: Model (blue, continuous line) vs Data (red, dashed line).

\begin{tabular}{|c|c|c|}
\hline \multicolumn{3}{|l|}{ (a) Pension System } \\
\hline & \multicolumn{2}{|c|}{ Average 2001-2007 } \\
\hline & Data & Model \\
\hline Pension Expenditure to GDP (PP/Y) \% & 6.54 & 6.65 \\
\hline (PP/Y: Old-age pensions) \% & 5.05 & 5.08 \\
\hline (PP/Y: Survival pensions) $\%$ & 1.49 & 1.56 \\
\hline Number of Retirement Pensions (millions) & 4.67 & 4.52 \\
\hline \multicolumn{3}{|l|}{ (b) Labor Supply } \\
\hline & \multicolumn{2}{|c|}{ Average 2001-2007 } \\
\hline & Data & Model \\
\hline Aggregate participation rate & 67.2 & 66.3 \\
\hline Female participation rate (Low education) & 38.1 & 41.6 \\
\hline (Medium education) & 61.6 & 62.8 \\
\hline (High education) & 76.5 & 67.7 \\
\hline
\end{tabular}

Table 3: Model performance in non-calibrated dimensions 

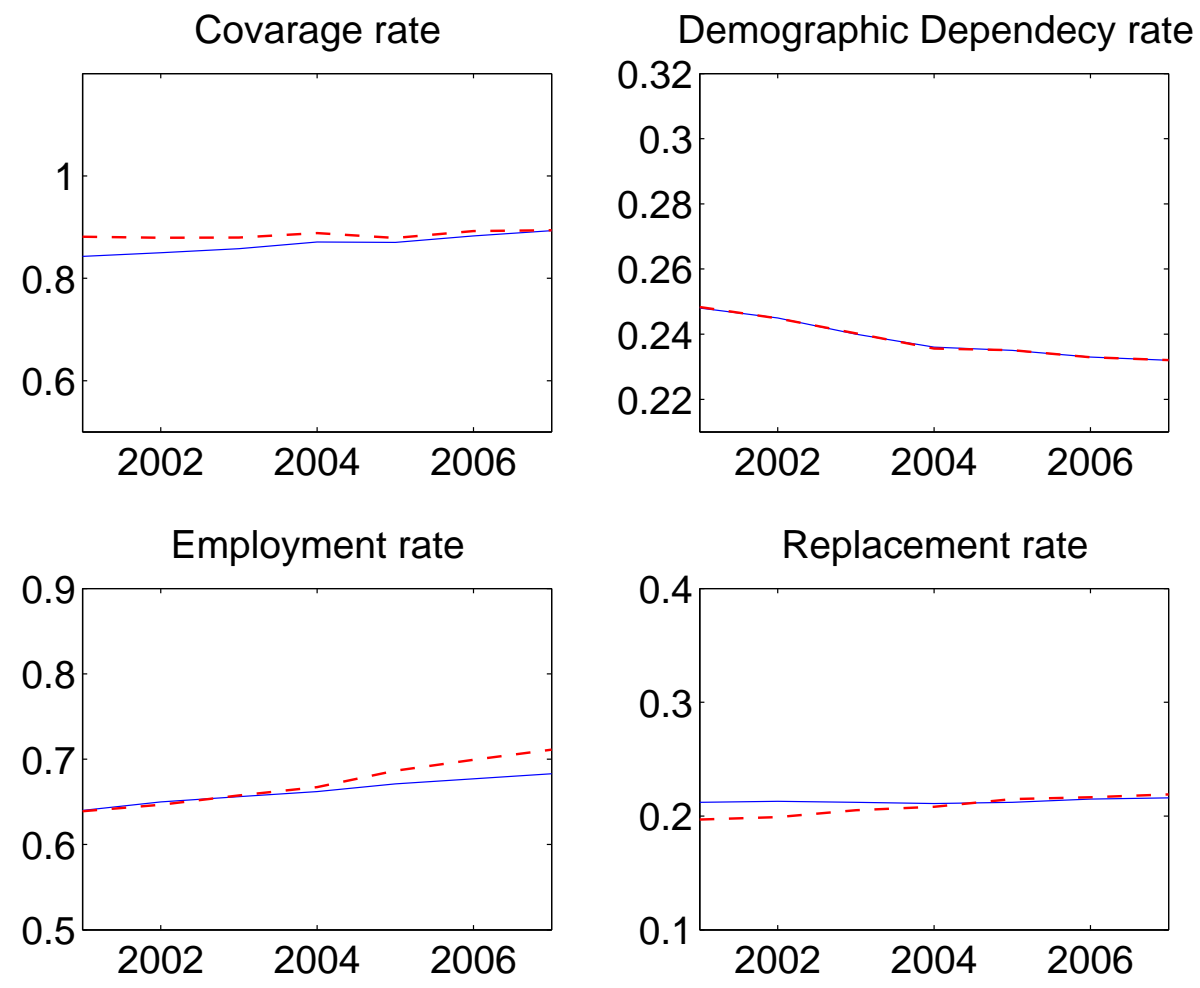

Figure 2: Breakdown of pension expenditure: Model vs reality performance in 2001-2007. Model (blue, continuous line) vs Data (red, dashed line).

Our stylized model of the pension system slightly underestimates the number of pensions and overestimates the replacement rate, especially at the beginning of the simulation. But the differences are small. Panel (a) in table 3 provides a few more details on these differences: the total number of old-age pensions is around $3 \%$ smaller than in the data and we generate a slightly inflated total expenditure in survival pensions.

The adjustment in terms of labor market performance is also good. The time-series of our simulated participation rates fails to reproduce the overheated labor market observed in the second half of the decade, but that is expectable. The differences by education are slightly larger (panel (b) in table 3) but clearly reflect the strong negative correlation between participation and education that we observe in the data. Finally, the bottom-right panel of figure 1 shows that we systematically underestimate the average retirement age. Given the relatively small number of different households at each posible age, the fine-tuning of this variable is not possible. Overall, we find the discrepancies of the simulated model and the data small enough to proceed to undertake a policy analysis with our model.

\section{Simulation Results}

In this section we present the results obtained after simulating the model of the previous sections in three different institutional environments. In section 4.1 we discuss the main properties of our benchmark economy, representing a projection of the Spanish economy 

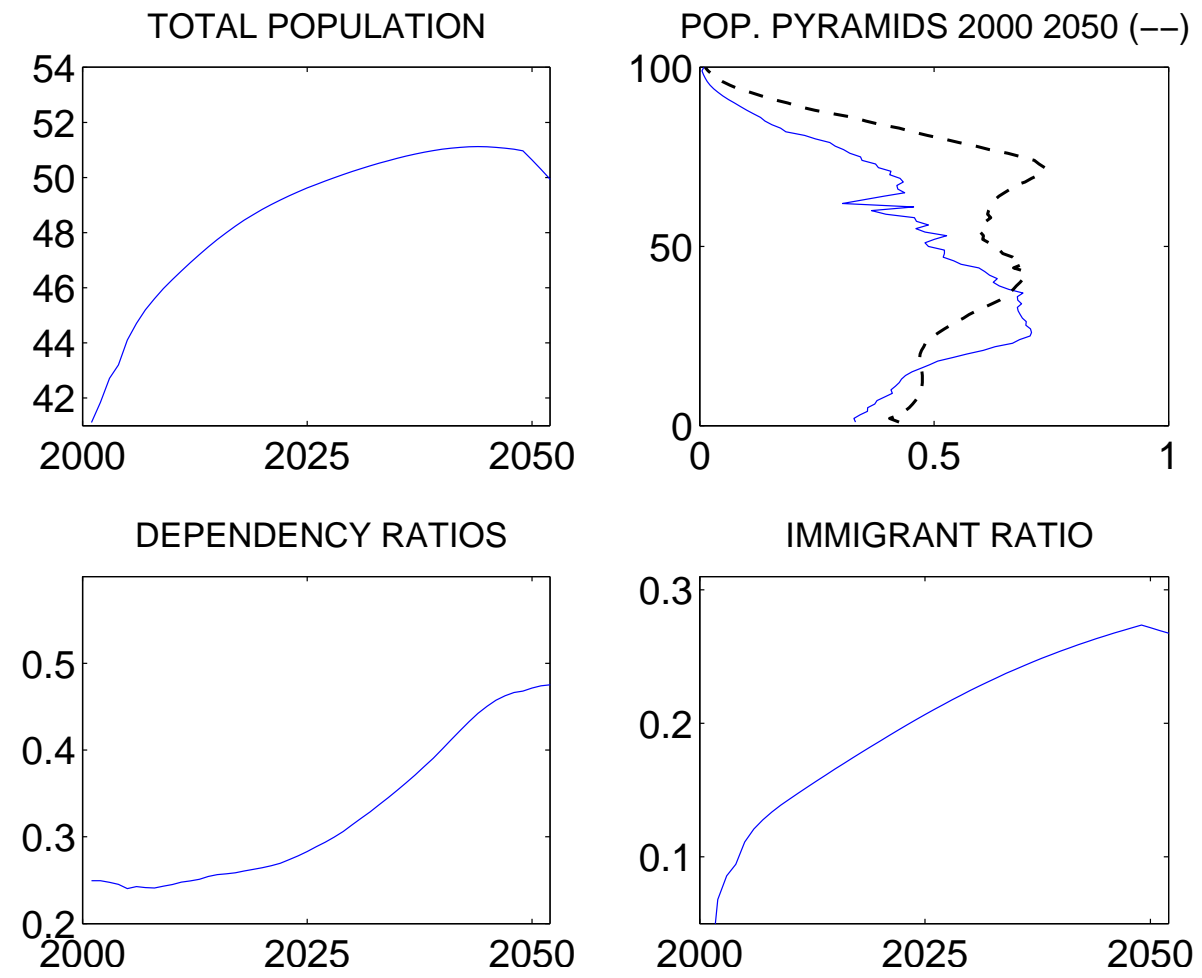

Figure 3: Demographic simulation: total population; age distribution of the population in 2000 and 2050; Dependency ratio and immigrant share of the total population.

with the current pension system. Section 4.2 discusses the effectiveness of the implemented reforms by exploring counterfactual simulations aimed at replicating the pension system in place before the 1997/2001 reforms. Finally, in section 4.3 we compare our benchmark results with the findings in an environment lacking survival pensions by construction.

\subsection{Projection in the benchmark}

\section{Demographic projections: an ageing population}

Figure 3 summarizes our projection of the main demographic variables for the first half of the 21st century, which, unsurprisingly, does not differ appreciably from the projections made by other institutions. ${ }^{30}$ The intensity of the immigration flows is large enough to fuel a rather dramatic increase in the absolute size of the Spanish population (top-left panel of figure 3). As a result, the share of first-generation immigrants grows to an astonishing $25 \%$ of the total population (bottom-right panel of figure 3). This phenomenon alleviates the ageing process of the population at large (as the distribution by age of the immigration flows is younger than that of the general population). However, as the bottom-left panel of figure 3 makes it clear, they fall short of stopping it altogether. The dependency ratio (defined as the ratio of the number of people older than 65 to those between 20 and 64) almost doubles in the

\footnotetext{
${ }^{30}$ Like the aforementioned INE projections or those in the latest EU Ageing report by the European-Comission (2009).
} 
interval 2000/2050 (from 26.9 to $51.2 \%$ ). The intensity of the ageing process can also be appreciated in the change in the shape of the population pyramid in the top-right panel of figure 3. These demographic patters underlie all the simulations that follow.

\section{The Economy Equilibrium path}

\begin{tabular}{cccccc}
\hline \hline Year & K/Y & Income tax rate $\varphi$ & PP/Y & Retirement age & Participation Rate \\
\hline 2001 & 2.379 & 12.74 & 6.94 & 62.67 & 64.00 \\
2005 & 2.428 & 16.89 & 6.47 & 62.70 & 67.10 \\
2010 & 2.523 & 15.31 & 7.03 & 62.13 & 69.30 \\
2015 & 2.609 & 15.82 & 7.41 & 62.10 & 72.00 \\
2020 & 2.686 & 16.03 & 8.27 & 62.08 & 73.80 \\
2025 & 2.754 & 16.36 & 9.68 & 62.12 & 75.00 \\
2030 & 2.800 & 17.36 & 11.43 & 62.19 & 75.90 \\
2035 & 2.815 & 18.89 & 13.33 & 62.28 & 78.00 \\
2040 & 2.806 & 20.89 & 15.82 & 62.54 & 79.10 \\
2045 & 2.745 & 21.96 & 17.18 & 62.75 & 79.50 \\
2050 & 2.669 & 21.73 & 17.82 & 62.75 & 79.90 \\
2055 & 2.642 & 21.49 & 17.81 & 62.61 & 79.00 \\
\hline \hline
\end{tabular}

Table 4: Macroeconomic aggregates in the benchmark simulation path

The projected evolution of the Spanish economy is represented by the blue continuous line in figures 4 to 7 and some key indicators in table 4 . The most important feature of the projection is the large increase in the pension expenditure, which is predicted to represent almost $18 \%$ of the annual GDP by 2050 (fourth column of table 4 ). This amounts to a $125 \%$ increase from the starting value, and is the result of expansions in both the old-age pensions and survival pensions (lower graphs of figure 4). At the same time population ageing has an impact of factor prices via changes in the capital to labor and capital to GDP ratios (K/Y is plotted on the top-left panel of figure 4 ). Labor becomes relatively more scarce than capital until around 2040, leading to slight improvements in wages versus interest rates.

As we keep the contribution rate constant, the increase in pension expenditure demands an adjustment of the income tax rate from 12.7 to $21.7 \%$ (see third column in table 4) to balance the overall public budget period by period. Note that the balance of the pension system alone plunges into deficit, as the top-right graph of figure 4 makes clear. ${ }^{31}$ Labor, pension and saving income are all taxed, implying that the extra fiscal burden is spread widely across the population. Household reaction is complex, due to opposite income and substitution effects. On the one hand, the net return on savings and working (even despite the gains in gross wages) is smaller, pushing towards more current consumption, earlier retirement and less female labor participation. On the other hand, a relatively lower life-cycle

\footnotetext{
${ }^{31}$ Other fiscal paths compatible with the fulfilment of a long-term budget constraint by the public sector are clearly possible. We opt for a period by period adjustment for two reasons. First, for simplicity: we avoid the complex intergenerational redistributional issues that would otherwise appear. Second, for realism. Spain has created a trust fund by saving some of the surplus generated in recent years, but its size is small (most of the surplus has actually been devoted to financing current expenditures). When the system shows a deficit, we doubt that Spain would convince international investors to finance significant delays in the adjustment schedule in absence of further reforms.
} 

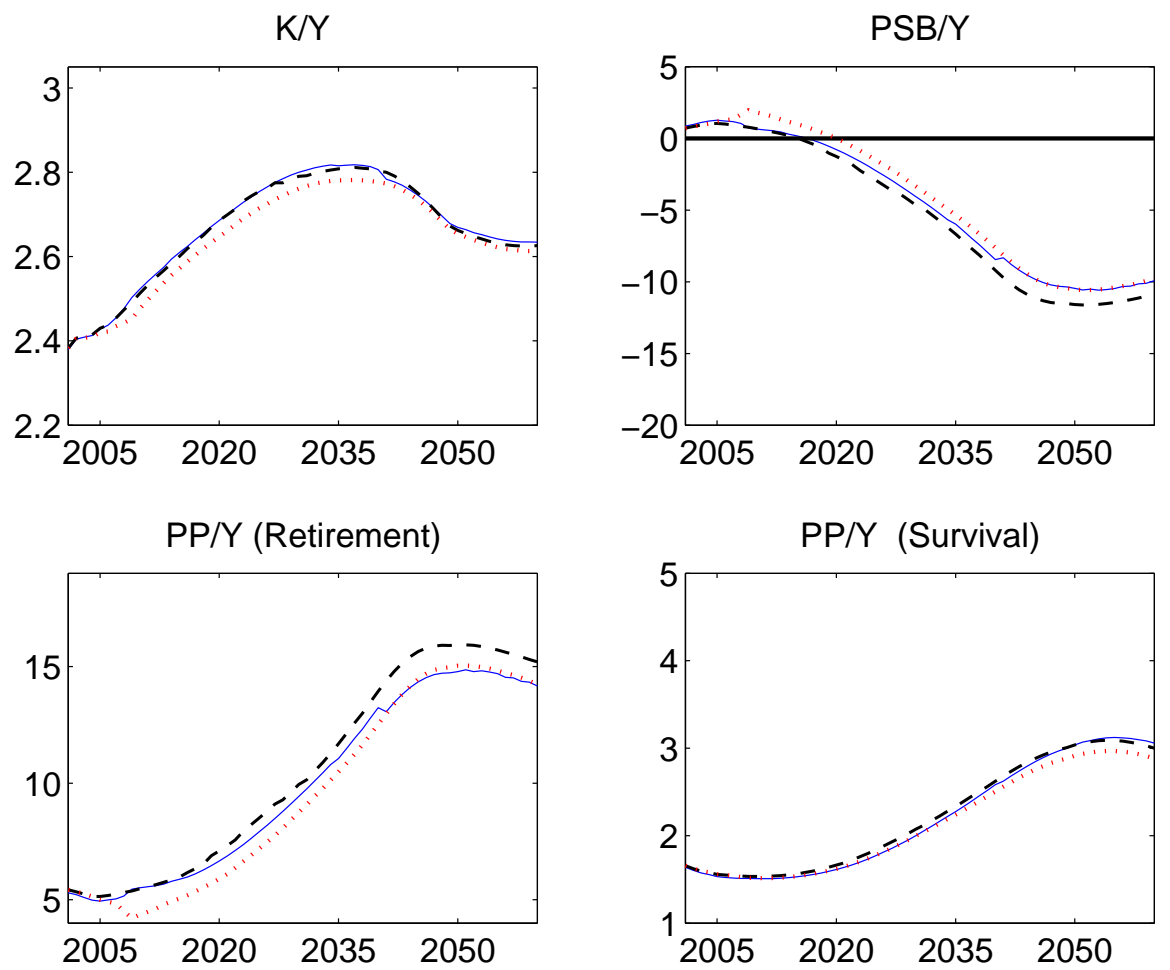

Figure 4: Simulated capital to output ratio and pension system's indicators in three institutional settings: Current (ie. benchmark simulation; blue continuous line (-)); Pre-1997/2001-reforms with early retirement (black dashed line (--)) and Pre-1997/2001-reforms without early retirement (red dotted line $(\cdot))$. PSB $/ \mathrm{Y}=$ pension system balance as a proportion of GDP; PP/Y=total pension expenditure to GDP by pension type (Old-age and survival).
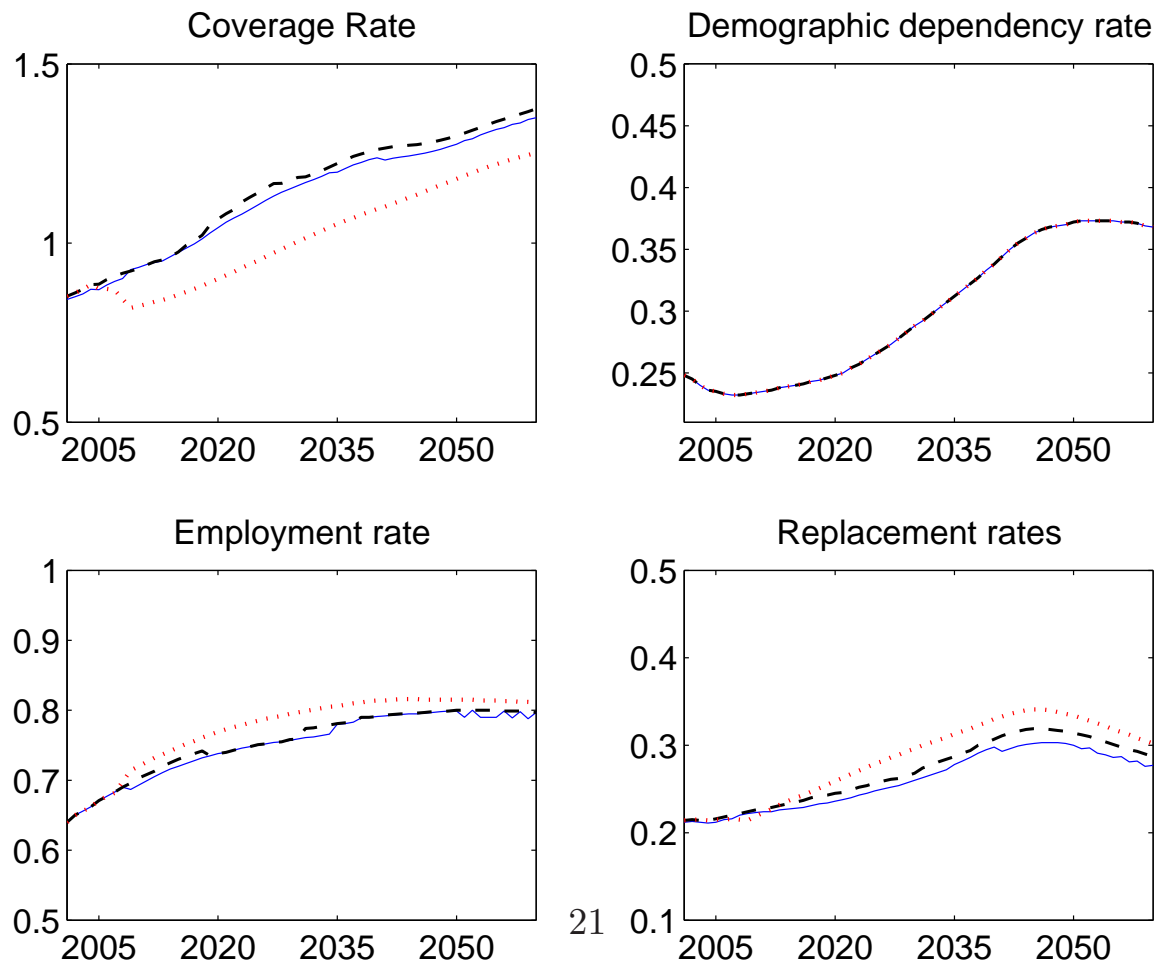

Figure 5: Pension Expenditure Decomposition (equation (13)) in three institutional settings: Current (ie. benchmark simulation; blue continuous line (-)); Pre-1997/2001-reforms with early retirement (black dashed line (--) ) and Pre-1997/2001-reforms without early retirement (red dotted line $(\cdot))$. 


\begin{tabular}{ccccc}
\hline $\begin{array}{c}\text { Averaging } \\
\text { period }\end{array}$ & $\begin{array}{c}\text { Early retirement } \\
\text { penalties }\end{array}$ & $\begin{array}{c}\text { Contribution } \\
\text { penalties }\end{array}$ & $\begin{array}{c}\text { Legal } \\
\text { ages }\end{array}$ & $\begin{array}{c}\text { Survival } \\
\text { pension }\end{array}$ \\
\hline$D=8$ & $\alpha_{0}^{E}=0.6$ & $\alpha_{0}^{H}=0.6$ & $\tau_{m}=60$ & $\alpha^{V}=0.45$ \\
& $\Delta \alpha_{+65}^{E}=0$ & $\Delta \alpha^{H}=2 \%$ & & \\
\hline
\end{tabular}

Table 5: Pension system parameters before the 1997/2001 reforms

wealth pushes in the opposite direction. With the current model calibration both effects are essentially balanced and the changes in the savings and participation rates are small. Only retirement shows a clear (although slight) tendency towards longer working careers (figure 6 ), especially among workers with higher education. ${ }^{32}$ Overall, the behavioral reaction to the demographic transition and its associated fiscal adjustment is very mild. In spite of this, the time series of the aggregate performance of the economy do display very evident modifications, mostly due to the progressive change in the composition of the population. For example, the average participation rates by education go appreciably up for most of the simulation (see figure 7), as a result of the replacement of older cohorts of workers with very low participation rates. Similarly, the increase in the aggregate average participation rate (top-left graph of figure 7) reflects both the generational replacement and the change in the distribution by education. All in all, our results confirm the qualitative findings in previous analysis of the Spanish economy (eg. Díaz-Giménez and Díaz-Saavedra (2009) o Sánchez (2010)). Section 4.3 below shows that the quantitative findings are, however, different.

The traditional explanation for the difficulties of PAYG pension systems emphasizes the role of demographic ageing. But a decomposition of the pension expenditure to GDP ratio along the lines of eq (13) reveals a very different picture (which can be clearly appreciated in figure 5). It is true that the dependency ratio contributes to higher expenses, but the coverage rate and the replacement rate also play very substantial roles. Again, these phenomena result from the long-term changes in the average participation rate by cohort and in the composition by education. More younger cohorts, with stronger labor market attachment, will result in a larger number of old-age pensions and, with some delay, of survival pensions. This increase reinforces the natural increase in survival pensions derived from an ageing population. The average pension will also grow faster than productivity. This reflects that historical pensions (formed in some cases according to different rules) are, on average, less generous than the most recent ones. It also reflects the impact of longer working careers (later retirement) in the individual pensions (ie. lower penalties). Note, finally, that the positive evolution of the labor market certainly helps to alleviate the increase in $\mathrm{PP} / \mathrm{Y}$. It is specially helpful in the first half of the simulation, a period with relatively large gains in the aggregate employment rates and, consequently, in output and contributions. In the second half, in contrast, the gains are much more moderate as the participation rate stabilizes. By then, the cohorts of new contributors progressively convert into larger numbers of new pensioners (increasing the coverage rate of the pension system). 


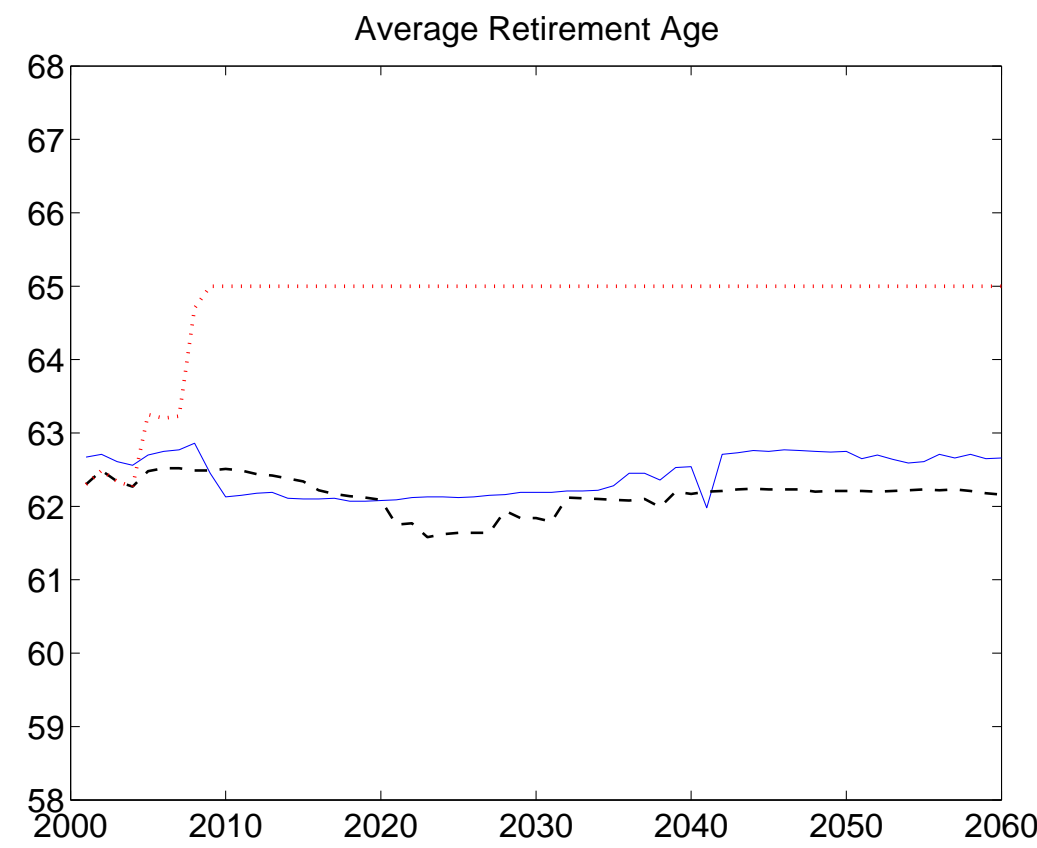

Figure 6: Simulated average retirement ages in three institutional settings: (i) Current (ie. benchmark simulation; blue continuous line (-)); Pre-1997/2001-reforms with early retirement (black dashed line $(--))$ and Pre-1997/2001-reforms without early retirement (red dotted line $(\cdot))$.
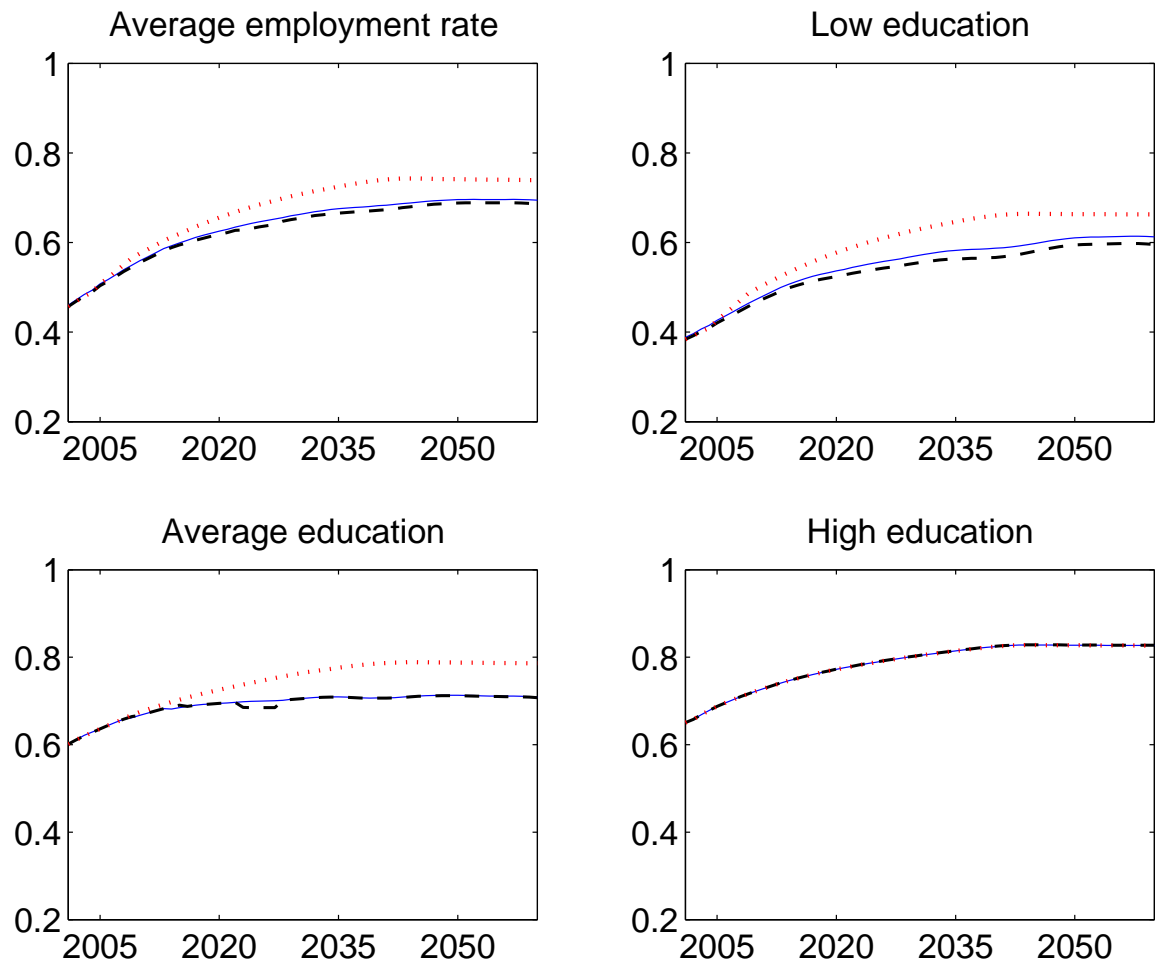

Figure 7: Simulated female participation rates in ¿mbree institutional settings: (i) Current (ie. benchmark simulation; blue continuous line $(-)$ ); Pre-1997/2001-reforms with early retirement (black dashed line $(--)$ ) and Pre-1997/2001-reforms without early retirement (red dotted line $(\cdot))$. 


\begin{tabular}{cccc}
\hline \hline & Current & Pre 1997, with ER & Pre 1997 \\
\hline IUL/Tax-Base \% & 105.4 & 114.3 & 94.2 \\
Average PP/Y & 11.93 & 12.60 & 11.35 \\
Maximum PP/Y & 17.82 & 18.97 & 17.95 \\
\hline \hline
\end{tabular}

Table 6: Pension expenditure 2010-2050 in three institutional environments: summary statistics. ER=Early Retirement IUL=Implicit Unfunded Liabilities of the pension system (present discounted value of the difference between present and future pensions and contributions to all cohorts alive in 2001, expressed as a proportion of the 2001 tax base). The discount factor is $5 \%$.

\subsection{Impact of the $1997 / 2001$ Reforms}

The current institutional environment is the result of several changes introduced in the pension rules during the interval $1997 / 2001 .^{33}$ As discussed in section 1, the reforms pursued two largely antagonist goals:

(i) Fiscal consolidation in the medium-run.

The government introduced changes in the pension formula aimed at reducing pension generosity. The most significant changes were (1) an increase in the length of the averageing period in the pension formula from $D=8$ to $D=15$ and (2) the imposition of stiffer penalties for retirement with a contribution record shorter than 25 years (set to $3 \%$ from an initial $2 \%$ )

(ii) Achieving a more egalitarian distribution of income.

This was pursued through the following three changes. First, the generosity of the survival pension was enhanced by an increase in the associated replacement rate from $45 \%$ to $52 \%$. Second, the right to claim the old-age pension before the age of 65 was extended to all cohorts, independently of when they became affiliated for the first time to the Social Security. ${ }^{34}$ In compensation, the early retirement age was delayed to 61 . Finally, the penalties for early retirement were relaxed for workers with very long contributive records.

Table 5 reflects the pension parameter values in effect before the 1997 changes (compare with table 1). In order to disentangle the impact of all these changes we consider two alternative counterfactual institutional environments (both characterized by the parameters in table 5 but differing on the legal possibility of early retirement) and compare them with the benchmark economy.

\section{Economic performance under the Pre-1997 reform, with early retirement}

The first alternative pension system that we simulate reflects the parameters in place before 1997 except for the prohibition of early retirement at the age of 60 . The results are represented by a dashed black line in figures 4 to 7 . In addition, table 6 reports some summary

\footnotetext{
${ }^{32}$ The discontinuity that figure 6 exhibits in year 2040 is created when households comprised of highly educated spouses decide to delay retirement from 65 to 66 .

${ }^{33}$ There have been some other smaller changes that we abstract from on simplicity grounds.

${ }^{34}$ In Spain, early retirement was initially introduced as a temporary measure aimed at easing the painful process of industrial restructuring in the 80's. Workers who became affiliated to the social security later than 1967 were originally not allowed to retire before 65 .
} 
statistics of the level of pension expenditure reached in each of our simulations and tables 7 and 8 (in appendix D) report the change in individual pensions by cohort, education and gender.

The extension of the averageing period reduced the level of the individual old-age pension, but with a varying degree of success depending on the educational level. Highly educated workers suffered the largest reductions, with drops of around $8 \%$ inflicted on the cohorts of older active workers at the time the reform was implemented. ${ }^{35}$ The pensions of low educated workers, in contrast, were little affected and, besides, their effective income was protected by the minimum pension guarantee (whose value was left untouched by the reform). A side effect of the reform was that, by reducing the individual benefit base, it was also undermining the effort to increase the survival pension by increasing (from $45 \%$ to $52 \%$ ) its replacement rate over the very same benefit base. As can be appreciated in the bottom-right of figure 4 the expenditure to GDP ratio in this type of pensions is actually smaller in a very short period of time after the reform.

All in all, the reform reduced the total pension expenditure to GDP by, roughly, $3 / 4$ of a percentage point, because of a reduction in the average replacement rate (as intended) and also by reducing the coverage rate (figure 5). This latter effect results from a small delay in the average retirement (figure 6), essentially achieved by elevating the early retirement age form 60 to 61 . The changes induced in the participation rates of females were very small. The overall impact of the reform in the financial condition of the pension system is summarized in the change in its implicit unfunded liabilities (IUL). ${ }^{36}$ As illustrated in table 6 , the relief resulting from all the reforms other than the change in early retirement is quantified as a 9 percentage point reduction (from $114.3 \%$ to $105.4 \%$ ) in the unfunded liabilities of the system. This triggered some small macroeconomic gains in the form of a mild process of capital deepening.

To complete the analysis, we measure the change in welfare generated by the reform with an Equivalent Variation (EV) in life-cycle consumption. ${ }^{37}$ Figure 8 shows the calculated EV by cohort and educational level conditional on the female's labour market status in the original (Pre-Reformed) economy. ${ }^{38}$ The general patterns are clear: welfare losses concentrate on the cohorts of older active workers, especially those with higher education, and largely with independence of the employment status of the female. Younger cohorts fare better because they profit from the tax cuts and macroeconomic gains generated for longer periods. ${ }^{39}$ Within cohorts, low income workers benefit most, thanks to the protection provided by minimum pensions. The welfare losses among top educated cohorts can be as large as almost $2 \%$ of life-cycle consumption.

\footnotetext{
${ }^{35}$ The differences by education are generated by the different properties of their life-cycle profile of labor productivity. Recall figure 14 .

${ }^{36}$ The Implicit Unfunded Liabilities is the present discounted value of the difference between the current and future pensions and contributions of all cohorts alive in 2001, expressed as a proportion of the 2001 tax base.

${ }^{37}$ We compute the EV as the proportional shift in the life-cycle profile of household consumption (under the initial pension rules) needed to match the life-cycle utility enjoyed after the reform. A positive value, then, implies a welfare gain.

${ }^{38}$ Our welfare figures include the endogenous adjustment in retirement, but fail to capture the welfare improvement derived from altering, in response to the reforms, the labor status of the female. This is actually the case in a small number of cohorts born immediately after the reform (peaking at around $2.2 \%$ for low educated households and $0.5 \%$ for medium educated workers). These are all marginal households, in the sense that their utility cost of female participation is very close to the threshold. For those households, our EV values overestimate the losses and underestimate the gains derived from the reform.

${ }^{39}$ Younger cohorts fare better with the exception of the cohorts of already retired households, who benefit from the tax relief generated by the reform without suffering any pension cut.
} 


\section{Economic performance under the Pre-1997 reform}

To complete the evaluation of the implemented reforms we simulate the economy under the pension system in place before 1997. That includes the prohibition of claiming the pension benefit before the age of 65 for workers who became affiliated to the social security later than 1967. We refer to this environment as simply the Pre 1997 framework. The simulation results are represented by a dotted red line in figures 4 to 7 .

Unsurprisingly, the biggest difference observed with the previous simulations occurs in retirement behavior, as the cohorts of workers without the right to retire early choose to wait till the normal retirement age to leave the labor force. This automatically implies that the Pre 1997 environment exhibits lower coverage rates than the benchmark. At the same time it has higher replacement rates than the benchmark, due to the increased pension associated to later retirement and for the reasons discussed in the previous section (in relation to the other institutional changes introduced in 1997). Overall, the reform results in pension cuts for a majority of the new retirees, while, at the same time, the pension bill of low educated workers increases due to the combination of earlier retirement and minimum pensions. In the aggregate, these two opposite forces largely cancel out, although the latter effect is a bit more important and the total annual old-age pension expenditure expands after the reform until around 2040 (and then goes slightly down thereafter). Survival pensions are, by the end of the simulation period, slightly more expensive due to the increase in the replacement rate of the benefit base. Overall, the reform increases the average annual total pension expenditure in $2010 / 2050$ by around $1 / 2$ of a percentage point, and the total IUL from $94.2 \%$ of the tax base in the Pre 1997 scenario to $105.4 \%$ in the benchmark (see table 6 ). The reform produces two appreciable macroeconomic side-effects. First, there is a moderate process of crowding in of private capital, resulting from the stronger saving effort undertaken by a majority of households (those that see their pension cut by the reform). Secondly, the timing of the tax hikes induced by the population aging changes. Now there is a stronger fiscal burden at the beginning of the path and a slightly more moderate one after 2050. Finally, on the labour market side, female participation of low and medium educational groups is about 5 percentage points lower as a result of the extension of early retirement.

Figure 9 displays a more marked pattern of welfare redistribution than that resulting from a reform with basically constant legal ages. As the group that benefits most from the right to early retirement, low income workers stand out as the big winners (especially in households with a double-earner, who tend to bring forward retirement in larger numbers). Remarkably, all the other households alive at the time of reform experience welfare losses (this time including already-retired workers). Future cohorts suffer progressively less severe losses and eventually benefit from the reform, again in inverse order to their educational attainment. 

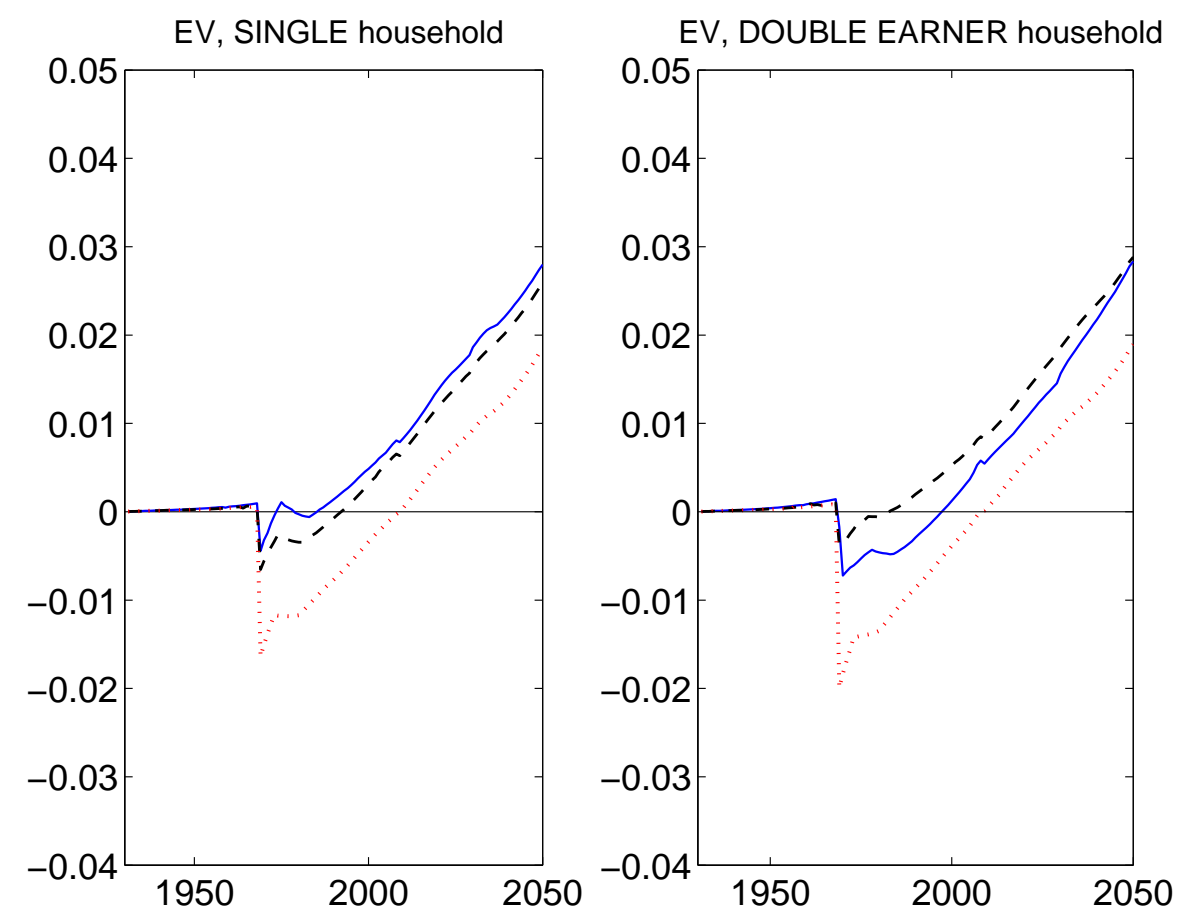

Figure 8: Equivalent Variation associated with the 1997-2001 reforms (assuming the pre 1997 system allowed for the early retirement of all cohorts) by education level: low (blue continuous line $(-)$ ), medium (black dashed line $(--)$ ) and high (red dotted line $(\cdot)$ ). A positive value implies a welfare gain after the reform.

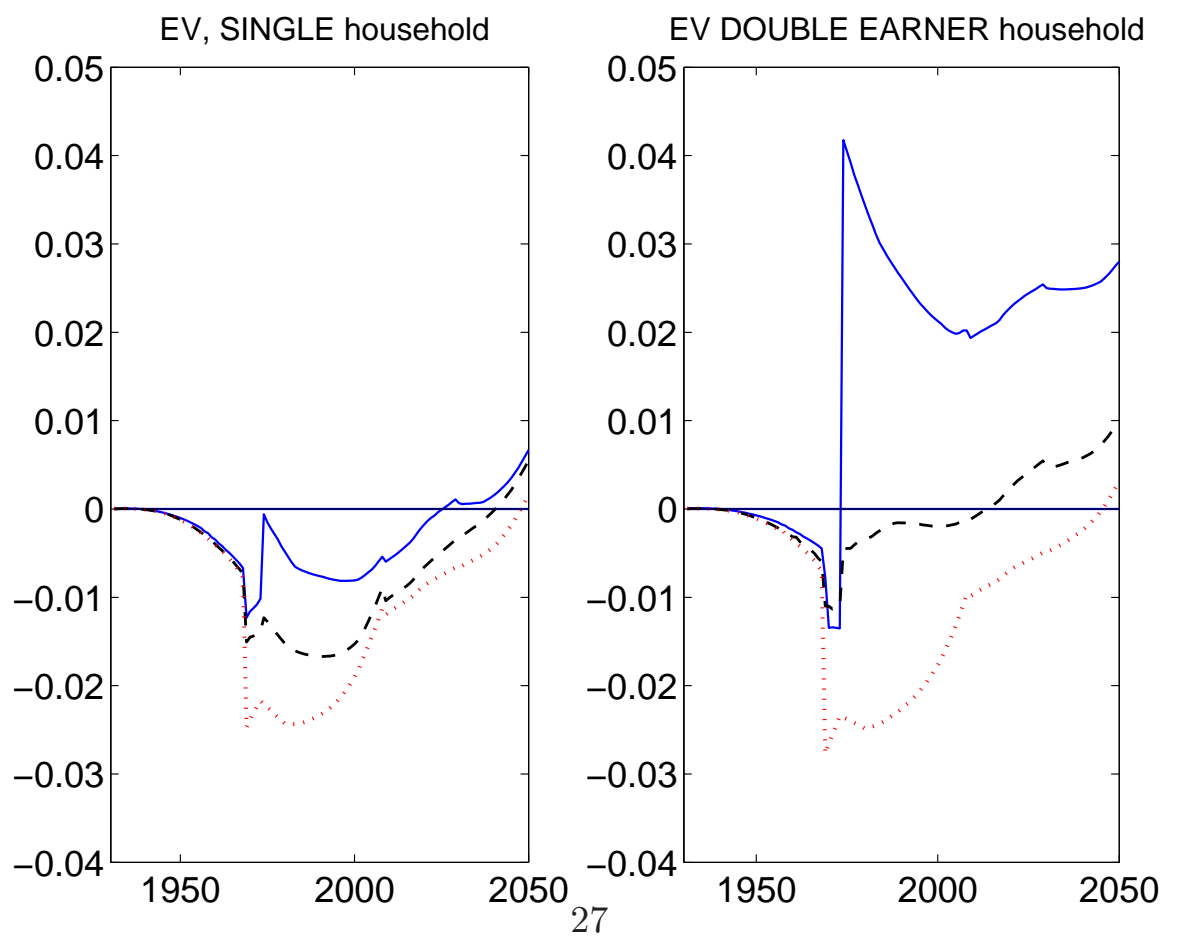

Figure 9: Equivalent Variation associated with the full 1997-2001 reforms by education level: low (blue continuous line $(-)$ ), medium (black dashed line $(--)$ ) and high (red dotted line $(\cdot)$ ). A positive value implies a welfare gain after the reform. 


\subsection{The importance of modelling survival pensions}

As we emphasized in section 1, up to now, the standard modeling framework employed in the pension literature has featured individuals rather than households. In a typical case, the individuals in the model are given a pension benefit whose value is set according to the regulations for old-age pensions in the country analyzed. Survival pensions and other transfers based on family relations are customary ignored (or, at best, added in present value terms to the old-age benefit). This modelling strategy makes perfect sense for a large number of research questions. However, we doubt that this is a satisfactory framework when projecting the aggregate pension system of a country. Survival pensions are granted according to their own specific rules which, in general, are very different to the old age rules. As a consequence, the level and dynamics of aggregate pension expenditure has to be different. It may well be the case, however, that the quantitative difference is small and the error incurred when using the standard model is acceptable.

In this section we assess this issue for the Spanish case with a simple experiment: to mimic standard models we assume that the old-age pension is the only one available and disregard any other transfer based on family links. Survival pensions are, therefore, ignored while keeping all the other ingredients of the model unaltered. To make the new simulation quantitatively meaningful we recalibrate the model aiming to reproduce the pension to GDP expenditure in the interval 2001-2007. This effort involves three changes: (1) inflating the initial condition on old-age pension to represent both retirement and survival benefits; (2) changing the number of retirement pensions until the model reproduces the initial total coverage rate (which we do by increasing the number of pensions per employee, $\phi$ ); and (3) reducing the size of the average old-age pension until we reproduce the initial replacement rate of the data (which we do by increasing the wedges separating gross income and pensionable income, $\chi) \cdot{ }^{40}$ The simulated behaviour of the resulting economy is presented in figures 10 and 11 . Note that the new calibration slightly overestimates the initial coverage and replacement rate. Still, its long term predictions in terms of the aggregate pension to GDP expenditure fall extremely short of the predictions of the more complete model of the previous sections. The long term divergence in the predicted $\mathrm{PP} / \mathrm{Y}$ peaks at a value of more than 1.5 percentage points (or a $10 \%$ error in the estimated value for 2050 ). The discrepancy is the result of significantly different simulated paths for the total replacement rate (which is underpredicted in a largely systematic way) and the coverage rate (which is overpredicted after 2020). Overall, the omission of survival pensions leads to an appreciable underestimation of the size of the implicit liabilities (IUL) of the pension system (98.3\% vs $105.4 \%$ with survival pensions). In the light of these results, we believe that researchers should proceed with caution when using single-agent models for the prediction of long-term pension expenditures.

\section{Conclusions}

We use a dynamic General Equilibrium model calibrated to the Spanish economy over the period 2001-2007 to assess the impact of the most recent reforms of the pension system, those undertaken in 1997/2001. The reforms aimed at improving the financial prospects of the system as well as enhancing solidarity. They combined a set of institutional parameter changes with contradicting financial and welfare consequences. Our target in this paper is to assess those financial and welfare implications. We introduce a novelty in the type of

\footnotetext{
${ }^{40}$ The parameter values employed in the re-calibrated model are as follows: $\alpha^{V}=0, b m_{V}=0, \phi=1.6, \chi_{1}=0.2$ and $\chi_{2}=\chi_{3}=0.6$.
} 


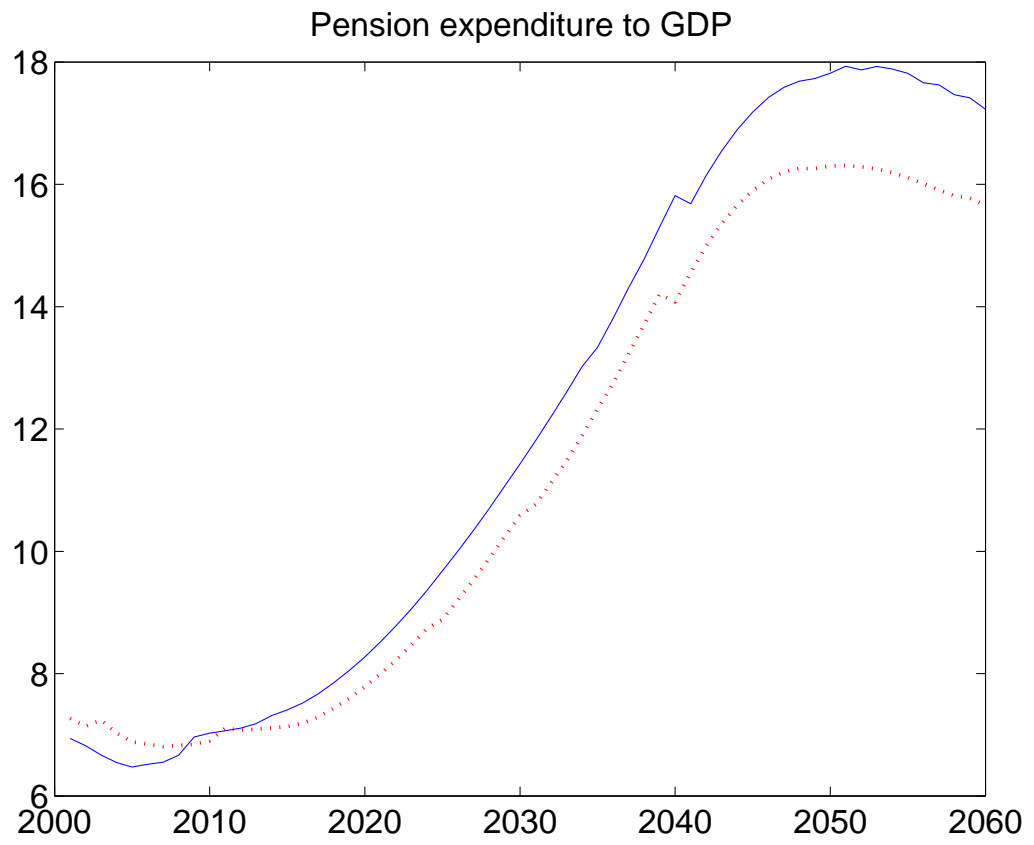

Figure 10: Pension expenditure to GDP ratio in the benchmark economy (blue continuous line) and in an economy without survival pensions (red dotted line $(\cdot)$ ).
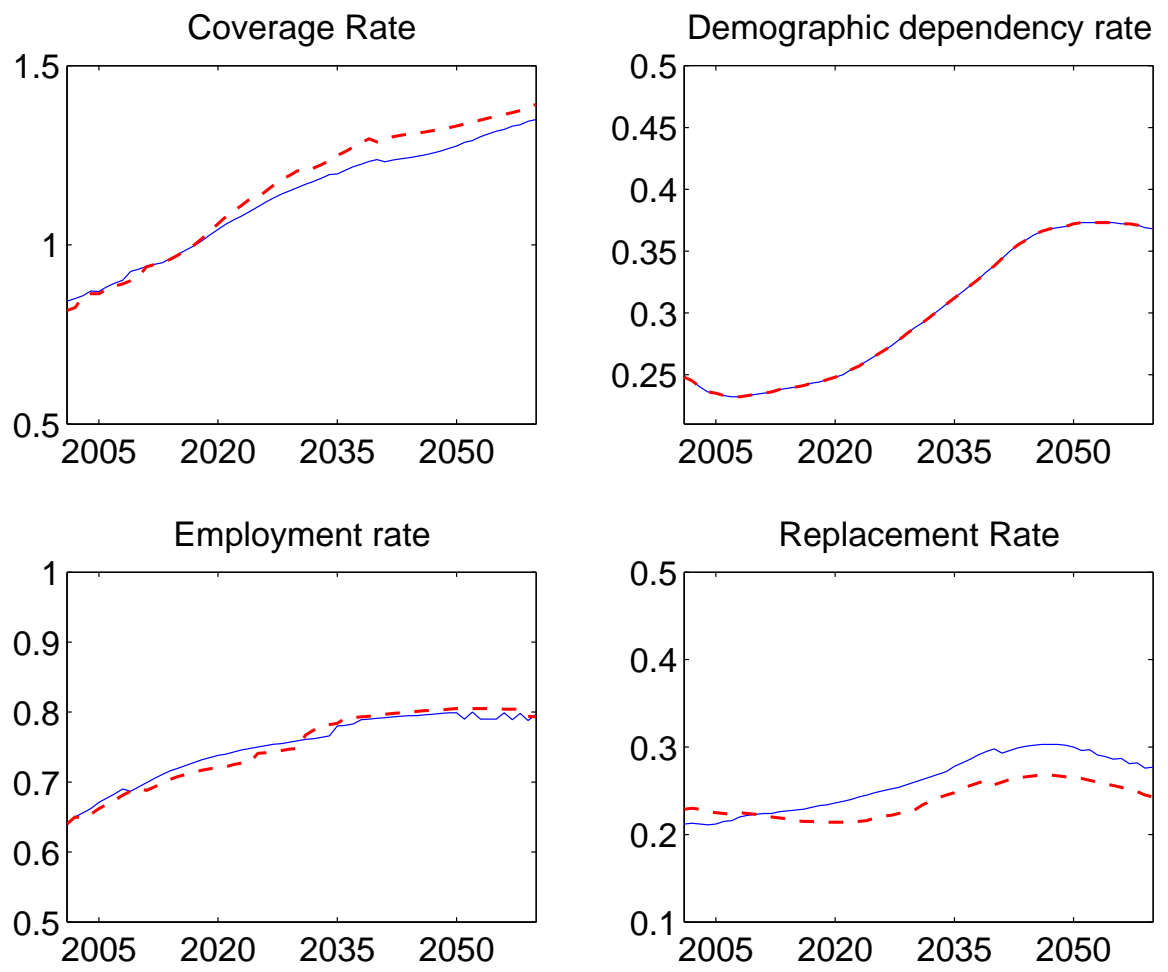

Figure 11: Pension Expenditure Decomposition (equation (13)) in the benchmark economy (blue continuous line) and in an economy without surivival pensions (red dashed line $(--)$ ). 
macroeconomic setup used to evaluate these issues in an ageing population economy. Instead of the standard single-earner household model, we use a two-potential-earners household model that allows some essential elements of the pension system (such as survival pensions and other rules contingent on household composition, like eg. minimum pensions) to be explicitly accounted for. Furthermore, we allow for the endogenous reaction of both female labour participation and of the retirement decisions of households.

Our main findings are as follows. First, like other studies in the literature, we conclude that the Spanish pension system is clearly unsustainable and pension expenditure will become about $18 \%$ of the GDP in 2050 (compared to the current $7 \%$ ). Survival pensions will also roughly double and so be responsible for $15 \%$ of the total increase. Second, the reforms implemented so far had an appreciable financial impact, but they were clearly ineffective in tackling the imbalances of the system: excluding the extension of the early retirement entitlement, the implemented reforms have reduced the implicit unfunded liabilities (as a proportion of the tax base) by around 9 percentage points; however, including the extension of early retirement, we find an increase of around 11 percentage points. Third, the reforms had significant welfare consequences by redistributing the cost of ageing against more educated individuals and in favor of less educated individuals. Also, they are detrimental for current cohorts of workers. Forth, as regards labour supply reactions, the reforms have had a small impact on female labour supply, but a significant impact on the retirement decision of low educated workers. Finally, we show that the omission of survival pensions from the analysis leads to a $7 \%$ underestimation of the implicit unfunded liabilities of the current system.In this sense, we think that our contribution goes beyond the particular figures obtained for the Spanish case, by showing that the projections of pension expenditures with standard, singleearner households models that abstract from survival pensions may deliver biased estimates of implicit pension burden in PAYG systems.

More generally, we believe that models with two-potential-earner households provide a fruitful framework for a more detail exploration of questions relating to pension and population ageing. For example, they may be specially well suited to studying the impact of pension reforms on poverty at advanced ages, on the labour supply behaviour (especially retirement and female participation decisions) and on the economic effects of increasing longevity. We do, in fact, think that it will be hard to address these matters without an explicit modelling of the heterogeneity within the household. 


\section{References}

Adam, Paula (1996). Mothers in an insider-outsider economy: the puzzle of Spain. Journal of Population Economics, 9, 301-323.

Attanasio, Orazio, Hamish Low, and Virginia Sanchez-Marcos (2008). Explaining changes in female labor supply in a life-cycle model. American Economic Review, 98, 1517-42.

Auerbach, A. J. and L. J. Kotlikoff (1987). Dynamic Fiscal Policy. Cambridge University Press.

Banks, James, Richard Blundell, and María Casanova (2007). The dynamics of retirement behavior in couples: reduced-form evidence from England and the US. Mimeo.

BBVA-Ivie, Fundación (2005). Stock de capital en España y su distribución territorial (19642002). Technical report.

Boldrin, Michele, Sergi Jiménez, and Franco Peracchi (2004). Micro-modelling of retirement behaviour in Spain. In Jonathan Gruber and David Wise (Eds.), Social Security programs and retirement around the world, pp. 499-578. NBER: University of Chicago Press.

Casanova, M (2009). Happy together: A structural model of couples' joint retirement choices. Mimeo.

Caseay, B, H Oxley, E Whitehouse, P Antolin, R Duval, and W Leibfritz (2003). Policies for an ageing society: recent measures and areas for further reform. Economics department working papers 369, OECD.

Caucutt, Elizabeth, Nezih Guner, and John Knowles (2002). Why do women wait? Matching, wage inequality and the incentives for fertility delay. Review of Economic Dynamics, 5, $814-855$.

Conesa, JC and C Garriga (2001). Sistema fiscal y reforma de la seguridad social. Working papers in Economics 67, Universitat de Barcelona. Espai de Recerca en Economia.

Consumer, Revista (2005). Siguen faltando plazas en las guarderías públicas, Revista Consumer Eroski, no. 91.

Cubeddu, L (1996). The intragenerational redistributive effects of Social Security. Working Paper 168, Universitat Pompeu Fabra.

INECSE, Instituto Nacional de Evaluación y Calidad del Sistema Educativo (2004). Evolución de las tasas de escolarización en las edades de niveles no obligatorios. Sistema estatal de indicadores de la educación.

De Nardi, MC, S İmrohoroğlu, and T. Sargent (1999). Proyected US demographics and Social Security. Review of Economic Dynamics, 2, 575-615.

Díaz-Giménez, J and J Díaz-Saavedra (2009). Delaying retirement in Spain. Review of Economic Dynamics (12), 147-167.

EPC (2006). The impact of ageing on public expenditure: projections for the EU25 member states on pensions, health care, long-term care, education and unemployment transfers (2004-2050). Special report 1/2006, Economic Policy Committee of the European Comission (DG ECFIN).

European-Comission (2009). 2009 ageing report: Economic and budgetary projections for the eu-27 member states (2008-2060). Technical Report 2, European Comission, Directorate General of Economic and Finacial Affairs (DG ECFIN).

Greenwood, J., A. Sehadri, and M. Yorukoglu (2005). Engines of liberation. Review of Economic Studies, 72, 109-133. 
Guner, Nezih, R. Kaygusuz, and G. Ventura (2008). Taxes, aggregates and the household. Mimeo.

Gustman, Alan and T Steinmeier (2000). Retirement in dual-career families: a structural model. Journal of Labor economics, 18, 503-545.

Gutiérrez-Domenech, María (2005). Employment after motherhood: a european comparison. Labour Economics (12), 99-123.

Hong, Jay H. (2008). Life insurance and the value of spouses: labor supply vs. household production. Mimeo.

Huggett, Mark and Gustavo Ventura (1999). On the distributional effects of Social Security reform. Review of Economic Dynamics (2), 498-531.

Hurd, Michael D. (1989). Mortality risk and bequest. Econometrica, 57, 779-813.

İmrohoroğlu, A., S. İmrohoroğlu, and D. Joines (1995, June). A life cycle analysis of Social Security. Economic Theory, 6, 83-114.

Jiménez, Sergi, JM Labeaga, and M Martínez (1999). Health status and retirement decisions for older european couples. Iriss working paper series 1999-01, IRISS at CEPS/INSTEAD.

Jiménez-Martín, Sergi and Alfonso R Sánchez-Martín (2007). An evaluation of the life-cycle effects of minimun pensions on retirement behavior. Journal of Applied Econometrics (22), 923-950.

Kaygusuz, R. (2008). Social Security with tow-earner households. Mimeo.

Kaygusuz, R. (2009). Taxes, aggregates and the household. Forthcoming in Review of Economic Dynamics.

Kotlikoff, L., K Smetters, and J Walliser (2000). Distributional effects in a general equilibrium analysis of Social Security. In M Feldstein (Ed.), The Distributional effects of Social Security reform. NBER: University of Chicago Press.

MTAS (2005). Informe de estrategia de España en relación con el futuro del sistema de pensiones. Technical report, Ministerio de Trabajo y Asuntos Sociales.

Olivetti, Claudia (2006). Changes in women's hours of market work: The effect of changing returns to experience. Review of Economic Dynamics, 9, 557-587.

Puch, Luis and Omar Licandro (1997). Are there any special features in the Spanish business cycle? Investigaciones Economicas, XXI, 361-394.

Rojas, JA (2005). Life-cycle earnings, cohort size effects and Social Security: a quantitative exploration. Journal of Public Economics, 89, 465-485.

Sánchez, Alfonso R (2010). Endogenous retirement and public pension system reform in Spain. Economic Modelling (27), 336-349.

Scholz, J K, A Seshadri, and S Khitatrakun (2006). Are americans saving optimally for retirement? Journal of Political Economy, 114, 607-643.

Storesletten, K., C. Telmer, and A. Yaron (1999). The risk sharing implications of alternative Social Security arrangements. Carnegie-Rochester Conference Series on Public Policy, 50, 213-259.

Tobío, C. (2003). Políticas públicas y estrategias privadas: la conciliación familia-empleo en España. Cambridge, MA: Servicio de Estudios de BBVA.

Whiteford, P. and E. Whitehouse (2006). Pension challenges and pension reforms in OECD contries. Oxford Review of Economic Policy, 22, 78-94. 


\section{APPENDIX}

\section{A Formal definition of equilibrium}

An equilibrium path over the time interval $\mathcal{T}$ consists of the following objects:

- Time series of the aggregate number of households $\left\{\mathcal{P}^{t}\right\}$ and their distribution by age, education and labour status of the female, $\mathcal{P}_{i j e}^{t}$ for all $i \in \mathcal{I}, \quad j \in J, \quad e \in$ $\{0,1\}, \quad t \in \mathcal{T}$. Note that, for each cohort and education, the distribution according to the labour status of the female is determined by a threshold on the utility cost, $\bar{z}_{i j}^{t}$, that is determined according to eq (14). The utility cost itself is assumed to be normally distributed.

- Assignments of consumption and asset holdings $\left\{c_{i j e}^{t}, a_{i j e}^{t}\right\}$ for all cohorts alive in $t \in \mathcal{T}$ and for all educational levels $j \in J$, and female participation situation $e \in\{0,1\}$.

- Time series of the inputs employed by the competitive firms of the economy $\left(K^{t}, L^{t}\right) t \in \mathcal{T}$

- A Public Policy consisting of the time series of taxes, public expenditure, minimum pensions (old-age and survival), maximum old-age pensions and maximum contributions:

$$
\left\{\varphi^{t}, C P^{t}, b m^{t}, b m V^{t}, b M^{t}, C_{M}^{t}\right\} \quad t \in \mathcal{T}
$$

- A price system: $\left\{r^{t}, w^{t}\right\} \quad t \in \mathcal{T}$

such that the following properties apply:

1. Endogenous population dynamics

Population aggregates and distributions are coherent with our demographic model, given the exogenous patterns specified for fertility, mortality, immigration flows and education distribution.

2. Rational behaviour by the households.

Household decisions are optimal (ie. solve problem (9)) given the price system and the public policy.

3. Clearance of the markets for capital and labour.

The capital and labour effectively employed by firms come from the aggregation of the household savings and labour supply:

$$
L^{t}=A^{t} H^{t} \quad H^{t}=\sum_{j=1}^{J} \sum_{i=20}^{\tau_{j}-1} \sum_{e=0}^{1} \mathcal{P}_{i j e}^{t} l_{i j e}^{t} \quad K^{t}=\sum_{j=1}^{J} \sum_{i=20}^{I-1} \sum_{e=0}^{1} \mathcal{P}_{i j e}^{t} a_{i j e}^{t}
$$

Where $l$ is the household supply of efficient labour units: $l_{i j e}^{t}=\pi_{i, 1} \varepsilon_{i j 1}^{t}+\pi_{i, 2} \varepsilon_{i j 2}^{t} I(e=1)$ (with $\pi$ and $\varepsilon$ are defined as in section 2.3 and $I($.$) an indicator function).$

4. Competitive prices:

$$
r+\delta=\frac{\partial F}{\partial K}\left(K^{t}, L^{t}\right) \quad w^{t}=\frac{\partial F}{\partial H}\left(K^{t}, L^{t}\right)
$$

5. Balanced Public budget:

$$
F I^{t}\left(\varphi^{t}\right)+P S B^{t}=C P^{t}
$$


where the public expenditure is a fixed proportion of aggregate output $C P^{t}=c_{-} p Y^{t}$; the fiscal income, $F I^{t}$, and the income from bequest, $B I^{t}$, takes the form:

$$
\begin{gathered}
F I^{t}=\varphi^{t} \sum_{j=1}^{J} \sum_{i=\tau_{j}}^{I} \sum_{e=0}^{1} \mathcal{P}_{i j e}^{t} i n c_{i j e}^{t}+B I^{t} \\
B I^{t}=\sum_{j=1}^{J} \sum_{i=20}^{I-1} \sum_{e=0}^{1}\left(1-h s_{i, j}^{t-i}\right) \mathcal{P}_{i j e}^{t-1} a_{i+1 j e}^{t-1}
\end{gathered}
$$

where inc stands for the total income of the household: $i n c_{i j e}^{t}=H I_{j i e}^{t}+r^{t} a_{i, j e}$, and $h s_{i, j}$ is the household survival probability (ie, the probability of survival of at least one of its members).

The pension system balance is given by

$$
P S B^{t}=C O T^{t}-P P^{t}
$$

where $P P^{t}$ and $C O T^{t}$ stand for the aggregate pension expenditures and the aggregate social contributions:

$$
P P^{t}=\sum_{j=1}^{J} \sum_{i=\tau_{j}}^{I} \sum_{e=0}^{1} \mathcal{P}_{i j e}^{t} H I_{j i}^{t} \quad C O T^{t}=\sum_{j=1}^{J} \sum_{i=1}^{\tau_{j}} \sum_{e=0}^{1} \mathcal{P}_{i j e}^{t} \cot _{i j e}^{t}
$$

With cot standing for the social contributions paid by the household, $\cot _{i j e}^{t}=\varsigma\left(\pi_{i, 1}^{t} \operatorname{cov}_{i, j, 1}^{t}+\pi_{i, 2}^{t} \operatorname{cov}_{i, j, 2}^{t} I(e=1)\right)$

6. Aggregate feasibility

$$
Y^{t}+(1-\delta) K^{t}+B I^{t}=K^{t+1}+B I^{t+1}+\sum_{i=20}^{I} \sum_{j=1}^{J} \sum_{e=0}^{1} \mathcal{P}_{i j e}^{t} c_{i j e}^{t}+C P^{t}+C C^{t}
$$

Where the child-care cost depends on the number of children and female wages by cohort and education: $C C^{t}=\sum_{i=20}^{24} \sum_{j=1}^{J} \mathcal{P}_{i j 1} C_{i}^{u+i-1}$

\section{B Economic and demographic calibration}

\section{B.1 Demographic Transition}

Figure 12 illustrates the changing patterns of fertility, mortality and immigration flows assumed in the simulations.

\section{B.2 Education}

The education classification explained in section 3.2.1 is chosen to mimic as closely as possible the distribution implemented in the European Community Household Panel (ECHP), from where we estimate the household earnings by education and gender. However, the historical evolution of labour market participation was taken from the Labor Force Survey whose large sample size allows a disaggregate analysis by cohort, gender and education. The classification in both samples are not identical: the Labor Force Survey has a more detailed description 
Fertility; 2001 (-) 2050(--)
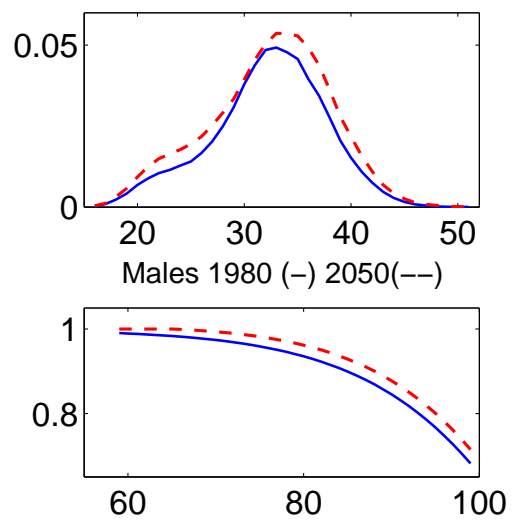

Life Expectancy at birth

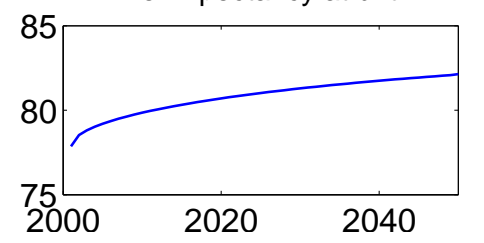

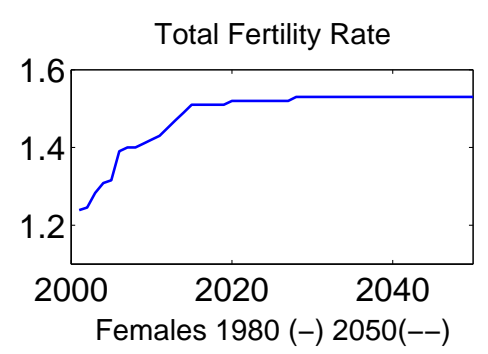
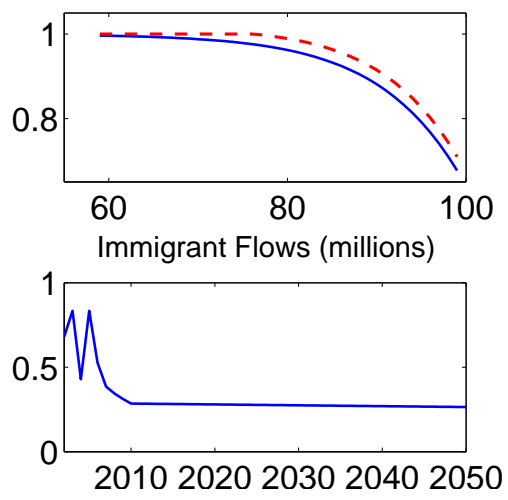

Figure 12: Simulated demographic transition

of the individual educational level (ie. with more categories). We, therefore, reclassified individuals according to the (more aggregated) categories in the ECHP.

\section{B.3 Life cycle profiles of efficiency labour units}

The data source to estimate individual life cycle profiles of efficiency labour units is the European Community Household Panel (ECHP) 1994-2000. We estimate a regression of the $\log$ of deflated annual gross earnings (this is variable PI110 in the ECHP: total income from work) on age and age squared. Note that, as we do not formally include unemployment in the model, we make our measure of earnings reflects the observed income losses created by unemployment episodes along the workers lifetime. In the regression we use married individuals (heads or spouses) aged 25 to 62 and include a linear trend as a regressor for compatibility with our assumption of constant, labour-augmenting, productivity growth. Separate regressions are estimated for each educational level and gender. In the simulations, the productivity in the age range 62/68 is obtained via extrapolation from the regression. In the interval $20 / 25$, in contrast, is assumed constant.

The estimated profiles are normalized taking the endowment of a low-educated female at the age of 20 as reference. The resulting profiles, reflecting the relative endowment of efficiency labour units of workers differing in education, gender and age (at the same calendar year) are shown in figure 14 . 


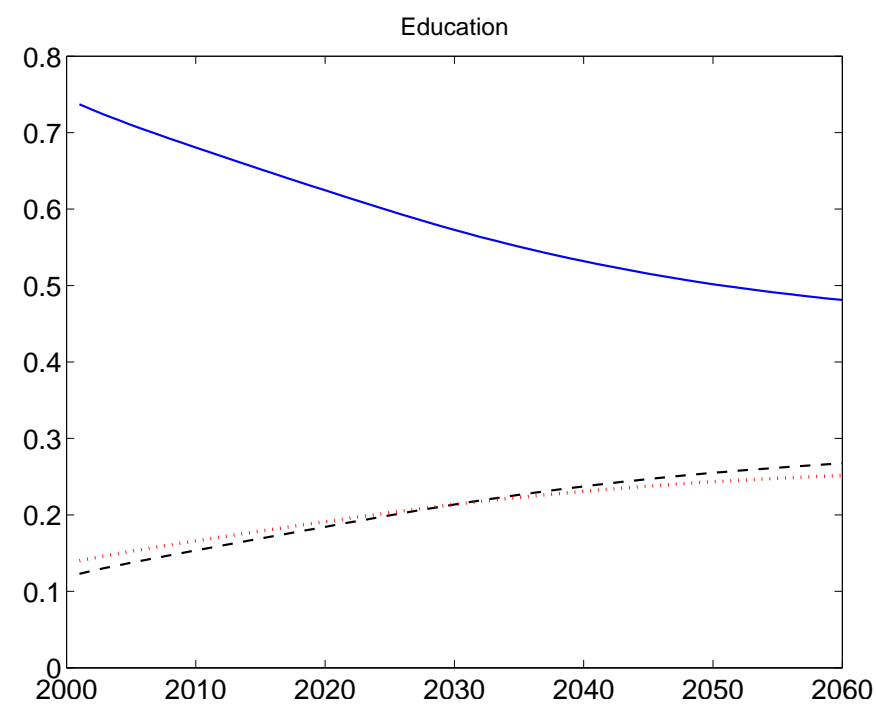

Figure 13: Simulated distribution by education. Time series of the proportion of households in each education level: low (blue continuous line $(-)$ ), medium (black dashed line $(--)$ ) and high (red dotted line $(\cdot))$

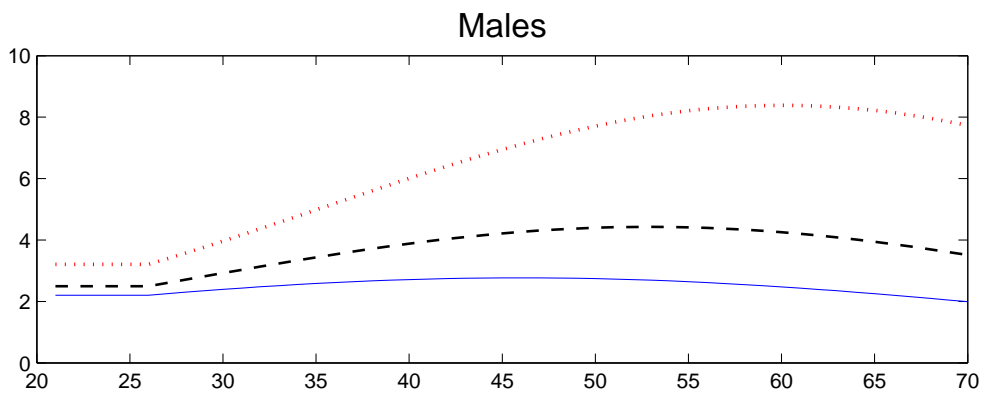

Females

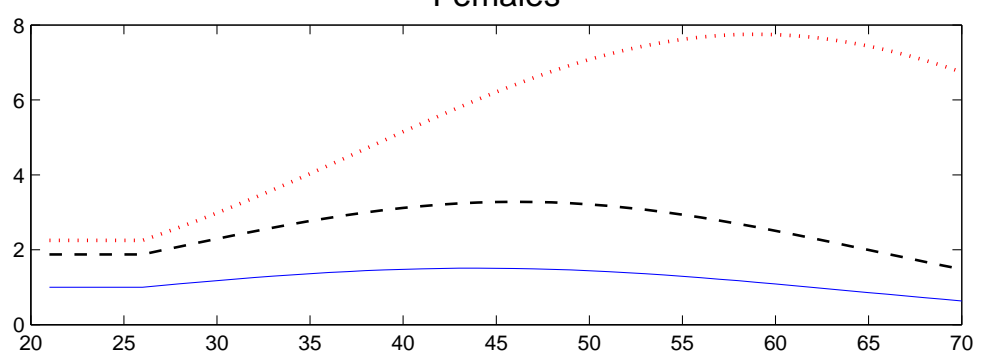

Figure 14: Life cycle profiles of efficiency labour units by gender and group of education: low (blue continuous line $(-)$ ), medium (black dashed line $(--)$ ) and high (red dotted line $(\cdot)$ ). All figures are ratios to the productivity of a low educated female at the age of 20 . 


\section{The female participation decision}

Under the assumptions in section 2.3 and 3.2 .3 , it is not difficult to express the life-cycle utility of single and two-earner households as, respectively:

$$
V(\tau)=U(Y) \Psi_{1}+\alpha \Psi_{2}(\tau) \quad V_{2}\left(\tau_{2}, z\right)=\left(U\left(Y_{2}\right)+z\right) \Psi_{1}+\alpha \Psi_{2}\left(\tau_{2}\right)
$$

where $U$ is the period utility function, $\tau$ and $\tau_{2}$ are the optimal retirement decisions for single and two-earner households; $Y$ and $Y_{2}$ are the life-cycle wealth in both cases and $\Psi_{i} i=\{1,2\}$ are constants that depends on the discount factor, survival probabilities and risk aversion. It is optimal of the female to participate in the labour market (i.e. $E=1$ ) when $V\left(\tau_{2}, z\right)>V(\tau)$. This optimal behaviour can be summarized by a threshold level $\bar{z}$ (the female of the household participates if $z<\bar{z}$ and stay out of the labour market otherwise). The formal definition of $\bar{z}$ is: $V(\tau)=V_{2}\left(\tau_{2}, \bar{z}\right)$. Linking the life-cycle wealth obtained in both cases in the following way:

$$
Y_{2}=(1+\gamma) Y \text { with } \gamma=\text { growth rate of } \mathrm{LC} \text { wealth after joining the labour force }
$$

we can derive the formal expression of the threshold:

$$
\bar{z}=\frac{Y^{1-\eta}}{1-\eta}\left[(1+\gamma)^{1-\eta}-1\right]+\alpha \Psi
$$

where $\Psi=\Delta \Psi_{2} / \Psi_{1}$ with $\Delta \Psi_{2}=\Psi_{2}\left(\tau_{2}\right)-\Psi_{2}(\tau)$.

This simple theory of female labour participation combines substitution effects (driven by the additional life-cycle income generated by female labour participation, $\gamma$, and the elasticity of substitution) and wealth effects (associated with the level of $Y$ ). With log-utility the threshold turns a simple function of $\gamma$ and the changed induced in retirement ages. Leaving aside the latter element (just for illustration purposes), we can informally describe how the model can reproduce the main stylized facts found in the data: (i) the higher participation rates of highly educated females observed in the crossed section would be the result of a larger $\gamma$ for those women (their added life-cycle labour income, net of participation costs, represents a larger proportion of the single-household $Y$ than for lower educated females); (ii) the trend towards higher participation rates by females of lower education implies that this difference is being progressively cut down for younger cohorts. The foundations of optimal retirement behaviour can be worked out in a similar way: see, for example, Jiménez-Martín and Sánchez-Martín (2007) where a continuous time version of the same model is explained in detail.

\section{Pension changes in the simulated reforms}

Tables 7 and 8 reproduce the changes in the individual pensions generated by the reforms, depending on gender, education and cohort. 


\begin{tabular}{ccccccc}
\hline \hline Cohort & \multicolumn{2}{c}{ Low Education } & \multicolumn{2}{c}{ Med Education } & \multicolumn{2}{c}{ High Education } \\
\hline & With ER & WITHOUT ER & With ER & Without ER & With ER & Without ER \\
1920. & 1 & 1 & 1 & 1 & 1 & 1 \\
1940. & 1 & 1 & 1 & 1 & 1 & 1 \\
1960. & 0.982 & 0.741 & 0.956 & 0.877 & 0.919 & 0.925 \\
1980. & 0.989 & 0.833 & 0.965 & 0.970 & 0.928 & 0.932 \\
2000. & 0.995 & 0.835 & 0.969 & 0.971 & 0.932 & 0.933 \\
2020. & 0.980 & 0.901 & 0.959 & 0.960 & 1.000 & 1.002 \\
\hline \hline
\end{tabular}

Table 7: Pension ratios for males: ratio of the average initial pension (by education and cohort) in the benchmark simulation (post reforms) to the average initial pension in the institutional setting before the 1997/2001-reforms (with and without Early Retirement, ER).

\begin{tabular}{ccccccc}
\hline \hline Cohort & \multicolumn{2}{c}{ Low Education } & \multicolumn{2}{c}{ Med Education } & \multicolumn{2}{c}{ High Education } \\
\hline & With ER & Without ER & With ER & Without ER & With ER & Without ER \\
1920. & 1 & 1 & 1 & 1 & 1 & 1 \\
1940. & 1 & 1 & 1 & 1 & 1 & 1 \\
1960. & 1.082 & 0.707 & 0.952 & 0.690 & 0.921 & 0.927 \\
1980. & 1.090 & 0.722 & 0.962 & 0.705 & 0.967 & 0.971 \\
2000. & 1.098 & 0.726 & 0.970 & 0.709 & 0.971 & 0.972 \\
2020. & 1.083 & 0.710 & 1.001 & 0.875 & 1 & 1.001 \\
\hline \hline
\end{tabular}

Table 8: Pension ratios for females: ratio of the average initial pension (by education and cohort) in the benchmark simulation (post reforms) to the average initial pension in the institutional setting before the 1997/2001-reforms (with and without Early Retirement, ER). 